\title{
Eigenfrequency constrained topology optimization of finite strain hyperelastic structures
}

\author{
Anna Dalklint ${ }^{1} \cdot$ Mathias Wallin $^{1} \cdot$ Daniel A. Tortorelli ${ }^{2}$ \\ Received: 13 September 2019 / Revised: 18 February 2020 / Accepted: 24 February 2020 / Published online: 17 May 2020 \\ (C) The Author(s) 2020
}

\begin{abstract}
This paper incorporates hyperelastic materials, nonlinear kinematics, and preloads in eigenfrequency constrained densitybased topology optimization. The formulation allows for initial finite deformations and subsequent small harmonic oscillations. The optimization problem is solved by the method of moving asymptotes, and the gradients are calculated using the adjoint method. Both simple and degenerate eigenfrequencies are considered in the sensitivity analysis. A wellposed topology optimization problem is formulated by filtering the volume fraction field. Numerical issues associated with excessive distortion and spurious eigenmodes in void regions are reduced by removing low volume fraction elements. The optimization objective is to maximize stiffness subject to a lower bound on the fundamental eigenfrequency. Numerical examples show that the eigenfrequencies drastically change with the load magnitude, and that the optimization is able to produce designs with the desired fundamental eigenfrequency.
\end{abstract}

Keywords Topology optimization · Eigenfrequency optimization $\cdot$ Finite strain $\cdot$ Nonlinear hyperelasticity $\cdot$ Element removal $\cdot$ Degenerate eigenfrequencies

\section{Introduction}

Topology optimization is extensively used in industry to guide early design processes. Unfortunately, existing formulations for topology optimization are primarily restricted to stiffness optimization of linear elastic structures. To allow for broader applicability, other objectives and nonlinearities should be considered.

Several studies combine nonlinear elasticity with topology optimization. In the work of Bruns and Tortorelli (2001), finite strain Green-Venant elastic structures and compliant mechanisms were designed. Different structures undergoing nonlinear elastic deformations were optimized for stiffness metrics by Buhl et al. (2000). This subject

Responsible Editor: Ole Sigmund

Anna Dalklint

anna.dalklint@solid.lth.se

1 Division of Solid Mechanics, Lund University, Box 118, SE-22100 Lund, Sweden

2 Center for Design and Optimization, Lawrence Livermore National Laboratory, Livermore, CA, USA was revisited by Kemmler et al. (2005) where the contribution from geometrical nonlinearities was investigated, and by Wallin et al. (2018) where a neo-Hookean hyperelastic material model was used. Other examples of nonlinear structural topology optimization include the work of Sigmund (2001a, b) where electromechanical devices with geometrical nonlinearities were designed using multiple materials, and the more recent work of Ivarsson et al. (2018) where the objective was to design impact mitigating structures while considering the effect of transients and finite strain viscoplasticity. Bruns et al. (2002) designed structures exhibiting snap-through behavior by modifying the basic arc-length algorithm. A phase-field-based topology optimization formulation that incorporates multi-material structures and large deformations was developed by Wallin et al. (2015).

Few have investigated the influence of eigenfrequency constraints in the topology optimization of structures undergoing finite deformations. To the authors' knowledge, the work of Yoon (2010) is the only example, wherein the fundamental eigenfrequency optimization of prestrained structures was considered by utilizing an internal element connectivity parameterization in contrast to the standard density-based approach. 
Topology optimization of linear elastic structures subject to eigenfrequency constraints was first proposed by Díaz and Kikuchi (1992), where structural reinforcements were introduced to increase the fundamental eigenfrequency. Various optimization formulations that consider eigenfrequencies have subsequently been suggested, e.g. the maximization of the fundamental eigenfrequency as done by Pedersen (2000) or the maximization of the gap between two eigenfrequencies as performed by Du and Olhoff (2007). In the work of Niu et al. (2009), a two scale optimization method was presented to maximize the fundamental eigenfrequency of multiscale structures. A level set-based topology optimization formulation has been proposed by Allaire and Jouve (2005) to maximize the fundamental structural eigenfrequency.

Unlike the aforementioned examples, our topology optimization incorporates frequency constraints of hyperelastic structures that undergo large deformations. The frequencies are obtained by linearizing the governing equations about the deformed state. This results in an eigenvalue problem which is numerically similar to the conventional linear case, the only difference being that the stiffness depends on the deformation. A neo-Hookean model is used to model the material response, which implies that both geometrical and material nonlinearities are included, unlike the formulation proposed by Yoon (2010) which only considers the former. This choice is made to accurately capture the response during finite deformations, since the conventional St. Venant material model is only applicable to large deformation with small strain. For additional discussion on hyperelastic models and topology optimization, see Klarbring and Strömberg (2013).

As pointed out in e.g. Seyranian et al. (1994), Du and Olhoff (2007), and Thore (2016), the occurrence of degenerate eigenfrequencies is rarely avoided in optimization studies that consider eigenfrequencies. The treatment of degenerate eigenfrequencies in topology optimization of linear elastic structures is well documented, where the nondifferentiability of degenerate eigenfrequencies is generally resolved by only considering directional derivatives, cf. Seyranian et al. (1994). This formulation is extended here to incorporate geometrical and material nonlinearities.

An often encountered issue when performing eigenfrequency topology optimization is the presence of spurious eigenmodes localized in nearly void regions. These spurious eigenmodes stem from the ersatz material and SIMP-penalization schemes. To eliminate this issue, Du and Olhoff (2007) suggested a nonlinear interpolation of the volume fraction, such that the mass in low volume fraction elements rapidly tends to zero. Low volume fraction elements are also known to cause convergence problems in the Newton-Raphson iterations when performing nonlinear finite element analyzes as these elements become highly distorted. To combat this problem, Wang et al. (2014) proposed an energy interpolation scheme which models these elements via small strain theory. In this work, we circumvent both of the preceding issues by implementing an element removal method technique proposed by Bruns and Tortorelli (2003), whereby the low volume fraction elements are removed from the finite element discretization.

We formulate the optimization problem as a maximization of the stiffness subject to maximum volume and minimum fundamental eigenfrequency constraints. From an engineer's perspective, this formulation is more relevant than the case where the objective is the fundamental eigenfrequency.

A well-posed topology optimization problem is formulated by using restriction in which a minimum length-scale is imposed on the design via a filter. The method of moving asymptotes (MMA) scheme is used to update the design, where the required gradients are computed using the adjoint sensitivity analysis. Both simple and degenerate eigenfrequencies are considered when performing the sensitivity analysis. The numerical examples illustrate the influence of the eigenfrequency constraints on the finalized topology.

\section{Preliminaries}

In this work, tensors and vectors will be written in bold faced symbols, e.g. $\boldsymbol{C}$, and all finite element matrices will be denoted in bold sans serif symbols, e.g. K. Lowercase subscripts $e$ indicate quantities associated with finite element $\Omega_{o}^{e}=1, \ldots, n_{e}$, where $n_{e}$ denotes the number of elements in the discretization. The conventional finite element assembly operation is represented by $\mathbb{\sum}$.

Since finite deformations are present, we distinguish between the undeformed, reference configuration $\Omega_{o}$, and the deformed, current configuration $\Omega$. It is assumed that a smooth mapping $\varphi$, between the reference configuration and the current configuration exists, i.e. the position vector $\boldsymbol{X}$, of each particle in the reference configuration is connected to its position $\boldsymbol{x}$, in the current configuration via $\boldsymbol{x}=$ $\boldsymbol{\varphi}(\boldsymbol{X}, t)=\boldsymbol{u}(\boldsymbol{X}, t)+\boldsymbol{X}$, where $\boldsymbol{u}$ denotes the displacement field. The local deformation is defined by the deformation gradient $\boldsymbol{F}=\nabla_{o} \boldsymbol{\varphi}=\mathbf{1}+\nabla_{o} \boldsymbol{u}$, where $\nabla_{o}$ is the material gradient operator on $\Omega_{o}$ and $\mathbf{1}$ is the second-order identity tensor. The Jacobian representing the volumetric change is given by $J=\operatorname{det}(\boldsymbol{F})$ and it is assumed that the mapping $\boldsymbol{\varphi}$, satisfies $J>0$. The local deformation can also be described by the right Cauchy-Green deformation tensor $\boldsymbol{C}=\boldsymbol{F}^{T} \boldsymbol{F}$ and the Green-Lagrange strain tensor $\boldsymbol{E}=\frac{1}{2}(\boldsymbol{C}-\mathbf{1})$.

In absence of body forces, the equation of motion is

$\nabla_{o} \cdot(\boldsymbol{F S})=\rho^{o} \ddot{\boldsymbol{u}} \quad$ in $\quad \Omega_{o}$, 
where $\rho^{o}$ denotes the mass density in the reference configuration and the symmetric second Piola-Kirchhoff stress tensor $S$ is related to the Cauchy stress $\sigma$, via $S=$ $J \boldsymbol{F}^{-1} \boldsymbol{\sigma} \boldsymbol{F}^{-T}$. The boundary of the body in the reference configuration $\partial \Omega_{o}$, with the unit normal $\boldsymbol{n}^{o}$, consists of two complementary surfaces, $\partial \Omega_{o t}$ and $\partial \Omega_{o u}$, over which the traction $\boldsymbol{t}^{o}=\boldsymbol{F} \boldsymbol{S n ^ { o }}$, and the displacement $\boldsymbol{u}=\boldsymbol{\varphi}-\boldsymbol{X}=\overline{\boldsymbol{u}}$, are prescribed, respectively.

The starting point for the finite element formulation is the weak form, which is stated as finding the differentiable $\boldsymbol{u}$ that satisfies the boundary condition $\boldsymbol{u}=\overline{\boldsymbol{u}}$ on $\partial \Omega_{o u}$, such that

$$
\int_{\Omega_{o}} \rho^{o} \delta \boldsymbol{u} \cdot \ddot{\boldsymbol{u}} d v+\int_{\Omega_{o}} \delta \boldsymbol{E}: \boldsymbol{S} d v-\int_{\partial \Omega_{o t}} \delta \boldsymbol{u} \cdot \boldsymbol{t}^{o} d s=0,
$$

for all smooth virtual displacements that satisfy $\delta \boldsymbol{u}=\mathbf{0}$ on $\partial \Omega_{o u}$. The virtual Lagrangian strain $\delta \boldsymbol{E}$, introduced in Eq. 2, is defined as

$\delta \boldsymbol{E}(\boldsymbol{\varphi}, \delta \boldsymbol{u})=\frac{1}{2}\left(\left(\nabla_{o} \delta \boldsymbol{u}\right)^{T} \boldsymbol{F}+\boldsymbol{F}^{T} \nabla_{o} \delta \boldsymbol{u}\right)$.

\section{Constitutive model}

Our constitutive assumption uses the isotropic compressible neo-Hookean strain energy model in which the strain energy $U^{N H}$ is expressed as

$U^{N H}=\frac{1}{4} K\left(\left(J^{2}-1\right)-\ln \left(J^{2}\right)\right)+\frac{1}{2} G\left(J^{-2 / 3} \operatorname{tr}(C)-3\right)$,

where $K$ and $G$ in the limit of infinitesimal strain correspond to the bulk and shear moduli. Since we assume a hyperelastic material model, the second Piola-Kirchhoff stress tensor is obtained from the strain energy as

$\boldsymbol{S}=2 \frac{\partial U^{N H}}{\partial \boldsymbol{C}}=\frac{K}{2}\left(J^{2}-1\right) \boldsymbol{C}^{-1}+G J^{-2 / 3}\left(1-\frac{\operatorname{tr}(\boldsymbol{C})}{3} \boldsymbol{C}^{-1}\right)$.

The fourth-order stiffness tensor associated with Eq. 4 becomes

$$
\begin{aligned}
\boldsymbol{D}=2 \frac{\partial \boldsymbol{S}}{\partial \boldsymbol{C}}= & a_{1} \boldsymbol{C}^{-1} \otimes \boldsymbol{C}^{-1}+a_{2}\left(\mathbf{1} \otimes \boldsymbol{C}^{-1}+\boldsymbol{C}^{-1} \otimes \mathbf{1}\right) \\
& +a_{3}\left(\boldsymbol{C}^{-1} \underline{\otimes} \boldsymbol{C}^{-1}+\boldsymbol{C}^{-1} \bar{\otimes} \boldsymbol{C}^{-1}\right),
\end{aligned}
$$

where $a_{1}=K J^{2}+\frac{2 G}{9} J^{-2 / 3} \operatorname{tr}(\boldsymbol{C}), a_{2}=-\frac{2 G}{3} J^{-2 / 3}$ and $a_{3}=\frac{G}{3} J^{-2 / 3} \operatorname{tr}(C)-\frac{K}{2}\left(J^{2}-1\right)$, respectively. The dyadic products $\otimes$ and $\bar{\otimes}$ are defined such that $(\boldsymbol{A} \otimes \boldsymbol{T}): \boldsymbol{H}=$ $\boldsymbol{A} \cdot \boldsymbol{H}^{T} \cdot \overline{\boldsymbol{T}}^{T}$ and $(\boldsymbol{A} \bar{\otimes} \boldsymbol{T}): \boldsymbol{H}=\boldsymbol{A} \cdot \boldsymbol{H} \cdot \boldsymbol{T}^{T}$, where $\boldsymbol{A}, \boldsymbol{T}$ and $\boldsymbol{H}$ are arbitrary second-order tensors.

\section{Filtration and penalization}

As is usual in topology optimization, the binary valued material indicator function $c(\boldsymbol{X})=\{0,1\}$ is replaced by the continuous varying volume fraction design field $z(\boldsymbol{X}) \in[0,1]$ that is subsequently penalized so that $z \rightarrow c$. The use of $z$ instead of $c$ convexifies the design space making the optimization cost and constraint functionals differentiable. Unfortunately, this representation of the design still generates an ill-posed optimization problem resulting in so-called chattering designs ${ }^{1}$. To obtain a well-posed optimization problem, we impose a lengthscale restriction on $z$ via the filter proposed by Bruns and Tortorelli (2003), which is based on studies and proofs presented by Bourdin (2001) and Bruns and Tortorelli (2001). Thus, volume fraction field $z$ is replaced by the filtered volume fraction field

$v(\boldsymbol{T})=\frac{\int_{\Omega_{o}} \omega(\boldsymbol{X}-\boldsymbol{Y}) z(\boldsymbol{Y}) d v}{\int_{\Omega_{o}} \omega(\boldsymbol{X}-\boldsymbol{Y}) d v} \approx \frac{\sum_{s=1}^{n_{e}} \omega\left(\mathbf{X}_{e}-\mathbf{Y}_{s}\right) \mathbf{z}_{s}}{\sum_{s=1}^{n_{e}} \omega\left(\mathbf{X}_{e}-\mathbf{Y}_{s}\right)}$,

where $\boldsymbol{X} \in \Omega_{o}^{e}$. In our discretization, we assume $\mathbf{z}$ is parameterized to be piecewise uniform over the elements and hence so is $v$. The kernel function $\omega$ is a cone filter function emanating from the element $\Omega_{o}^{e}$ centroid position $\mathbf{X}_{e}$ and enveloping the surrounding element $\Omega_{o}^{s}$ centroid positions $\mathbf{Y}_{s}$ that lie within the filter radius $r$ of $\mathbf{X}_{e}$, cf. Bruns and Tortorelli (2003). The filter relation (7) can ultimately be expressed in discretized format as

$\mathbf{v}=\mathbf{z} \mathbf{z}$

where $\mathbf{Z}$ is the $\left[n_{e} \times n_{e}\right]$ filter matrix, $\mathbf{z}=\left[\mathrm{z}_{1}, \mathrm{z}_{2}, \ldots, \mathbf{z}_{n_{e}}\right]^{T}$ and $\mathbf{v}=\left[\mathrm{v}_{1}, \mathrm{v}_{2}, \ldots, \mathrm{v}_{n_{e}}\right]^{T}$ are the vectors of element volume fractions and filtered volume fractions.

Elements possessing $v_{e}(\boldsymbol{X})=1$ and $\mathrm{v}_{e}(\boldsymbol{X})=0$ indicate elements full and devoid of material, respectively. Unfortunately, the filtering produces gray regions consisting of elements for which $v_{e}(\boldsymbol{X}) \in(0,1)$. To reduce the extent of these regions, the SIMP scheme-based on the work by Bendsøe (1989) is used, wherein we replace $U^{N H}$ with

$U=\chi(v) U^{N H}$,

where

$\chi(v)=v^{p}\left(1-\delta_{0}\right)+\delta_{0}$.

Here $\delta_{0}$ represents a lower threshold in the ersatz material model which ensures the well-posedness of the elasticity problem and $p$ controls the level of penalization. It is clear that as $\chi(v) \rightarrow \delta_{0}$ the material mimics void, whereas as

\footnotetext{
${ }^{1}$ The chatter terminology is used by Young (2000) to describe an illposed optimal control problem in which the solution consists of a nonconverging sequence of infinitesimal pulse loads.
} 
$\chi(v) \rightarrow 1$ the material realizes the neo-Hookean strain energy function (4).

\section{Total Lagrangian FE formulation}

The displacement field $\boldsymbol{u}$ is interpolated in each finite element using the standard finite element polynomial shape functions $\mathbf{N}$, to define the displacement field $\mathbf{u}$, i.e. $\mathbf{u}(\boldsymbol{X})=$ $\mathbf{N}(\boldsymbol{X}) \mathbf{a}_{e}(t)$ where $\mathbf{a}_{e}(t)$ is a vector consisting of the nodal displacements for finite element $e$ at time $t$. Differentiating $\mathbf{u}$ gives the acceleration $\ddot{\mathbf{u}}(\boldsymbol{X})=\mathbf{N}(\boldsymbol{X}) \ddot{\mathbf{a}}_{e}(t)$, and virtual displacement $\delta \mathbf{u}(\boldsymbol{X})=\mathbf{N}(\boldsymbol{X}) \delta \mathbf{a}_{e}(t)$, fields. Using these approximations, the discrete virtual Lagrangian strain in Eq. 3 is expressed as

$\delta \mathbf{E}\left(\mathbf{a}_{e}, \delta \mathbf{a}_{e}\right)=\mathbf{B}\left(\mathbf{a}_{e}\right) \delta \mathbf{a}_{e}=\left(\mathbf{B}^{c}+\mathbf{B}^{l}\left(\mathbf{a}_{e}\right)\right) \delta \mathbf{a}_{e}$,

where $\mathbf{B}\left(\mathbf{a}_{e}\right)$ has been decomposed into constant $\mathbf{B}^{c}$, and linear $\mathbf{B}^{l}\left(\mathbf{a}_{e}\right)$ terms, cf. Crisfield (1993) for details ${ }^{2}$. Using the arbitrariness of the virtual nodal displacement and the finite element interpolations in Eq. 2 yields the finite element equation of motion

$\mathbf{M a ̈}+\mathbf{F}_{i n t}(\mathbf{a})-\mathbf{F}_{e x t}=\mathbf{0}$,

where the mass matrix $\mathbf{M}$, internal force vector $\mathbf{F}_{i n t}$, and external force vector $\mathbf{F}_{\text {ext }}$, are

$$
\begin{array}{r}
\mathbf{M}=\sum \int_{\Omega_{o}^{e}} \rho \mathbf{N}^{T} \mathbf{N} d v, \\
\mathbf{F}_{\text {int }}(\mathbf{a})=\sum \int_{\Omega_{o}^{e}} \mathbf{B}\left(\mathbf{a}_{e}\right)^{T} \mathbf{S} d v, \\
\mathbf{F}_{\text {ext }}=\sum \int_{\partial \Omega_{o t}^{e}} \mathbf{N}^{T} \mathbf{t}^{o} d s .
\end{array}
$$

In Eq. 13, we emphasize that $\rho=v \rho^{o}$.

\subsection{Displacement controlled Newton-Raphson}

In this work, we load the structure quasi-statically by imposing a displacement $\boldsymbol{u}=\overline{\boldsymbol{u}}$ on $\partial \Omega_{o u}$ to obtain the deformed configuration $\Omega$. We subsequently evaluate the natural frequencies on $\Omega$. The displacement controlled Newton-Raphson scheme is used to solve the quasi-static residual equilibrium equation obtained by neglecting inertia and equating $\mathbf{t}^{O}=\mathbf{0}$ in Eq. 12, i.e.

$\mathbf{r}_{f}(\mathbf{a})=\mathbf{F}_{i n t, f}(\mathbf{a})=\mathbf{0}$,

where the subscript $f$ refers to the equations associated with the unconstrained degrees of freedom, i.e. the

\footnotetext{
$\overline{{ }^{2} \text { Voigt notation }}$ is used to represent second- and fourth-order finite element tensors.
}

free displacements $\mathbf{a}_{f}$, as opposed to the prescribed displacements $\mathbf{a}_{p}$.

To solve (16), we employ the Newton-Raphson scheme where the residual (16) is linearized to obtain the unknown increment $d \mathbf{a}_{f}$, in each equilibrium iteration as the solution to

$\mathbf{r}_{f}(\mathbf{a})+\frac{\partial \mathbf{r}_{f}(\mathbf{a})}{\partial \mathbf{a}_{f}} d \mathbf{a}_{f}=\mathbf{0} \Rightarrow \mathbf{K}_{f f}(\mathbf{a}) d \mathbf{a}_{f}=-\mathbf{r}_{f}(\mathbf{a})$,

where $\mathbf{K}_{f f}$ is the partition of the tangent stiffness matrix

$\mathbf{K}(\mathbf{a})=\frac{\partial \mathbf{r}(\mathbf{a})}{\partial \mathbf{a}}=\sum \int_{\Omega_{o}^{e}}\left(\mathbf{B}^{T}\left(\mathbf{a}_{e}\right) \mathbf{D B}\left(\mathbf{a}_{e}\right)+\mathbf{G}^{T} \mathbf{H G}\right) d v$,

corresponding to the free degrees of freedom. In Eq. 18, G contains the gradient of the element shape functions, $\mathbf{H}$ is a symmetric block matrix that contains the stress, and $\mathbf{D}$ is the Voigt notation stiffness matrix that corresponds to $\boldsymbol{D}$. The explicit format of these matrices appears in e.g. Crisfield (1993).

\section{Eigenfrequency analysis}

The conventional eigenvalue problem is formulated for zero initial strain. In contrast to the conventional problem, our eigenvalue problem considers nonzero initial strain wherein the stiffness and eigenfrequencies depend on the displacement field.

Our structure is preloaded via the constant displacement $\mathbf{a}_{p}$ such that $\mathbf{r}_{f}=\mathbf{F}_{\text {int }, f}(\mathbf{a})=\mathbf{0}$ where $\mathbf{a}$ is the displacement field in the equilibrium configuration. To formulate the eigenvalue problem, we introduce a small time-dependent perturbation $\delta \mathbf{a}_{f}$ from $\mathbf{a}_{f}$ so that

$\mathbf{a} \rightarrow \mathbf{a}+\delta \mathbf{a} \Rightarrow \ddot{\mathbf{a}} \rightarrow \ddot{\mathbf{a}}+\delta \ddot{\mathbf{a}}=\delta \ddot{\mathbf{a}}$,

where $\delta \mathbf{a}_{p}=\delta \ddot{\mathbf{a}}_{p}=\mathbf{0}$. Insertion of Eqs. 19 into 12 results in

$\mathbf{M} \delta \ddot{\mathbf{a}}+\mathbf{F}_{i n t}(\mathbf{a}+\delta \mathbf{a})=\mathbf{0} \Rightarrow \mathbf{M}_{f f} \delta \ddot{\mathbf{a}}_{f}+\mathbf{F}_{i n t, f}(\mathbf{a}+\delta \mathbf{a})=\mathbf{0}$.

A Taylor series expansion of the internal force and ignoring higher-order terms yields

$\mathbf{F}_{i n t, f}(\mathbf{a}+\delta \mathbf{a})=\mathbf{F}_{i n t, f}(\mathbf{a})+\frac{\partial \mathbf{F}_{i n t, f}(\mathbf{a})}{\partial \mathbf{a}_{f}} \delta \mathbf{a}_{f}$,

which when combined with Eqs. 16 and 18 allows (20) to be expressed as

$\mathbf{M}_{f f} \delta \ddot{\mathbf{a}}_{f}+\mathbf{K}_{f f} \delta \mathbf{a}_{f}=\mathbf{0}$. 
As with the linear problem, we see that $\delta \mathbf{a}_{f}$ is a harmonic oscillation, i.e. $\delta \mathbf{a}_{f}=\sum_{j=1}^{n} \boldsymbol{\phi}_{j} e^{i \omega_{j} t}$ where $\left(\omega_{j}^{2}, \boldsymbol{\phi}_{j}\right)$ are the eigenpairs that are obtained from the real, symmetric generalized eigenvalue problem

$\mathbf{K}_{f f} \boldsymbol{\phi}_{j}=\omega_{j}^{2} \mathbf{M}_{f f} \boldsymbol{\phi}_{j}, \quad j=1, \ldots, n$,

where $n$ denotes the dimension of the problem, i.e. the number of free nodal degrees of freedom in $\mathbf{a}_{f}$, and $\omega_{j}^{2}$ are the square eigenfrequencies. In our study, the stiffness and mass matrices are symmetric, real, and positive semi-definite, and hence the eigenfrequencies are real and positive, cf. Clough and Penzien (1993). Subsequently, the eigenvalues are placed in ascending order such that $0<$ $\omega_{1}^{2} \leq \omega_{2}^{2} \leq \ldots \leq \omega_{j}^{2} \leq \ldots \leq \omega_{n}^{2}$, and their associated eigenmodes are mass-normalized such that

$\boldsymbol{\phi}_{j}^{T} \mathbf{M}_{f f} \boldsymbol{\phi}_{k}=\delta_{j k}, \quad j, k=1, \ldots, n$,

where $\delta_{j k}$ denotes the Kronecker's delta.

The formulation of the eigenvalue problem for a nonlinear system (23) is clearly similar to the one obtained in conventional analyzes, cf. e.g. Du and Olhoff (2007); however, it is emphasized that the eigenpairs of the nonlinear system depend on the equilibrium displacement field a since $\mathbf{K}_{f f}$ is a function of the displacement $\mathbf{a}$.

\section{Consideration of low volume fraction elements}

In our formulation, void regions are modeled using the minute volume fraction $\delta_{0}$ which is assumed to have a negligible influence on the structural response. Unfortunately, for eigenfrequency constrained finite deformation problems, the existence of low volume fraction elements is problematic.

Firstly, low volume fraction elements yield so-called spurious, localized eigenmodes. The issue arises when solving (23) since the mass scales linearly, i.e. $p=1$, with the volume fraction, whereas the stiffness scales at $p>1$, cf. Eqs. 10 and 13, which implies that the stiffnessto-mass ratio becomes very small in the void regions where $v(\boldsymbol{X}) \rightarrow 0$. Several ways to suppress these nonphysical eigenmodes have been suggested in the literature. By far the most common method, as proposed by Du and Olhoff (2007), weights the volume fraction such that the stiffnessto-mass ratio, i.e. the spurious eigenfrequency, becomes large.

Secondly, regions consisting of low volume fraction elements often become highly distorted in our finite deformation analysis. This causes convergence issues in the Newton-Raphson equilibrium iterations. Different means for addressing this issue have also been proposed, e.g. by introducing an interpolation of the strain energy such that void regions are modeled using small deformation theory as done by Wang et al. (2014), or by simply relaxing the convergence criterion of the Newton-Raphson iterative scheme as done by Pedersen et al. (2001).

To eliminate the aforementioned problems associated with low volume fraction elements, we employ the element removal method proposed by Bruns and Tortorelli (2003). This procedure is able to both remove and reintroduce the finite elements. Elements are removed from the analysis if their filtered volume fraction is smaller than a prescribed small threshold $\varepsilon_{r}>0$, i.e. if $\mathrm{v}_{e}<\varepsilon_{r}$; they are reintroduced if $v_{e}>\varepsilon_{r}$. The threshold $\varepsilon_{r}$ must be chosen such that the discontinuities in the gradient information due to the nonzero tolerance $\varepsilon_{r}$ do not greatly affect the optimization. We did not experience any numerical difficulties when using this method. More details regarding the implementation of this method are discussed in Bruns and Tortorelli (2003).

Despite using the element removal scheme, the remaining small regions with intermediate volume fractions $v(\mathrm{x}) \in$ $\left(\varepsilon_{r}, 1\right)$ gave rise to spurious eigenmodes due to the ersatz material model. To combat this, we follow Ferrari et al. (2018) and set the lower bound $\delta_{0}$ to a relatively large value $\delta_{0}=10^{-3}$. This choice of $\delta_{0}$ increases the magnitude of the spurious eigenfrequencies such that they do not obstruct the optimization. For most ersatz material models, a large $\delta_{0}$ leads to significant load-carrying capabilities of void regions which might adversely affect the resulting design. However, since we employ element removal, this problem is avoided as the effected regions are small.

By examining the evolution of the potential energy or the kinetic energy of the spurious modeshapes, i.e.

$$
\begin{aligned}
& E_{p o t}=\boldsymbol{\phi}_{j}^{T} \mathbf{K}_{f f} \boldsymbol{\phi}_{j}=E_{k i n}=\omega_{j}^{2} \boldsymbol{\phi}_{j}^{T} \mathbf{M}_{f f} \boldsymbol{\phi}_{j}, \\
& j=1, \ldots, n
\end{aligned}
$$

we verify the absence of low energy spurious eigenmodes in the nearly void interface regions. Indeed, such modes would be readily identified by low $E_{\text {pot }}$ values.

\section{Topology optimization}

The objective of our topology optimization is to distribute material to build a stiff structure with fundamental eigenfrequency greater than $\omega_{\min }$. Following Niu et al. (2011), the stiffness of a displacement loaded structure is defined by

$g_{o}=\mathbf{F}_{e x t, p}^{T} \mathbf{a}_{p}$

where $\mathbf{F}_{\text {ext, } p}$ contains the reaction forces of the degrees of freedom for which the displacements $\mathbf{a}_{p}$ are imposed. 
Constraints on the $n_{c}$ lowest eigenvalues are cast as

$g_{j}=\omega_{\text {min }}^{2}-\omega_{j}^{2} \leq 0, \quad j=1, \ldots, n_{c}$,

and on the maximum volume $V_{\max }$ as

$g_{V}=\int_{\Omega_{0}} v d v-V_{\max } \leq 0$.

The optimization problem together with the box constraints on the components of $\mathbf{z}$ is formulated as

$\mathcal{P}\left\{\begin{array}{l}\min _{\mathbf{z}}-g_{o} \\ \text { s.t }\left\{\begin{array}{l}g_{j} \leq 0, \quad j=1, \ldots, n_{c} \\ g_{V} \leq 0 \\ 0 \leq \mathbf{z}_{e} \leq 1, \quad e=1, \ldots, n_{e}\end{array}\right.\end{array}\right.$

where we reformulate the objective as a minimization problem and consider the three smallest eigenfrequencies, i.e. $n_{c}=3$.

\section{Sensitivity analysis}

The optimization problem is solved using the gradientbased MMA, where the sensitivities of the objective and constraint functions are computed using the adjoint approach. An example verifying the analytical sensitivities of the eigenfrequencies is found in the Appendix. In the subsequent sensitivity analysis, the nondifferentiable effect of the element removal scheme for elements with $v_{e}=\varepsilon_{r}$ is neglected. Fortunately, for small choices of $\varepsilon_{r}$, we did not experience any convergence issues during the optimization.

Since the problem is regularized using a filter, the sensitivities are obtained using the chain rule and Eq. 8 as

$\frac{\partial g_{i}}{\partial \mathbf{z}}=\left(\frac{\partial v}{\partial \mathbf{z}}\right)^{T} \frac{\partial g_{i}}{\partial v}=\mathbf{z}^{T} \frac{\partial g_{i}}{\partial v}$,

where we emphasize that $\mathbf{Z}$ is the constant filter matrix.

\subsection{Sensitivity analysis of objective}

The sensitivity of the objective function as defined in Eq. 26 has previously been outlined by Luo et al. (2017), but for completeness, it is summarized below. Using the adjoint method, we augment $g_{o}$ and write it equivalently as

$g_{o}=\mathbf{a}_{p}^{T} \mathbf{F}_{i n t, p}+\boldsymbol{\mu}^{T} \mathbf{F}_{i n t, f}$,

where we utilize $\mathbf{F}_{\text {int }, f}=\mathbf{0}$ and introduce the adjoint vector $\boldsymbol{\mu}$. Differentiation of Eq. 31 with respect to the filtered volume fraction variables yields

$$
\begin{aligned}
\frac{\partial g_{o}}{\partial v_{e}}= & \mathbf{a}_{p}^{T} \frac{\partial \mathbf{F}_{i n t, p}}{\partial v_{e}}+\boldsymbol{\mu}^{T} \frac{\partial \mathbf{F}_{i n t, f}}{\partial v_{e}}+ \\
& +\left(\mathbf{a}_{p}^{T} \frac{\partial \mathbf{F}_{i n t, p}}{\partial \mathbf{a}_{f}}+\boldsymbol{\mu}^{T} \frac{\partial \mathbf{F}_{i n t, f}}{\partial \mathbf{a}_{f}}\right) \frac{\partial \mathbf{a}_{f}}{\partial v_{e}} .
\end{aligned}
$$

Assigning the adjoint vector $\boldsymbol{\mu}$ such that

$\mathbf{K}_{f f} \boldsymbol{\mu}=-\mathbf{K}_{f p} \mathbf{a}_{p}$,

where $\mathbf{K}_{f p}=\frac{\partial \mathbf{F}_{i n t, f}}{\partial \mathbf{a}_{p}}$ annihilates the $\frac{\partial \mathbf{a}_{f}}{\partial v_{e}}$ contribution in Eq. 32. The sensitivity is thus reduced to

$\frac{\partial g_{o}}{\partial v_{e}}=\mathbf{a}_{p}^{T} \frac{\partial \mathbf{F}_{i n t, p}}{\partial v_{e}}+\boldsymbol{\mu}^{T} \frac{\partial \mathbf{F}_{i n t, f}}{\partial v_{e}}=\boldsymbol{\alpha}^{T} \frac{\partial \mathbf{F}_{i n t}}{\partial v_{e}}$,

where

$\boldsymbol{\alpha}=\left[\begin{array}{c}\boldsymbol{\mu} \\ \mathbf{a}_{p}\end{array}\right]$.

\subsection{Sensitivity analysis of simple eigenvalues}

If eigenfrequency $\omega_{j}$ satisfies $\omega_{j-1}<\omega_{j}<\omega_{j+1}$, the eigenpair $\left(\omega_{j}^{2}, \phi_{j}\right)$ is said to be simple and the corresponding normalized eigenmode $\phi_{j}$ is unique. The sensitivity of a simple eigenvalue with respect to the design variables of a linear elastic structure is trivially obtained as, cf. e.g. Haftka and Gürdal (1992)

$$
\frac{\partial \omega_{j}^{2}}{\partial v_{e}}=\boldsymbol{\phi}_{j}^{T}\left(\frac{\partial \mathbf{K}_{f f}}{\partial v_{e}}-\omega_{j}^{2} \frac{\partial \mathbf{M}_{f f}}{\partial v_{e}}\right) \boldsymbol{\phi}_{j}, \quad j=1, \ldots, n_{c} .
$$

Unfortunately, the corresponding sensitivity analysis for a nonlinear elastic body is less straight forward since the stiffness matrix depends on the displacement, i.e. $\frac{\partial \mathbf{K}}{\partial v_{e}} \rightarrow$ $\frac{\partial \mathbf{K}}{\partial v_{e}}+\frac{\partial \mathbf{K}}{\partial \mathbf{a}} \frac{\partial \mathbf{a}}{\partial v_{e}}$. To eliminate the implicitly defined sensitivity $\frac{\partial \mathbf{a}}{\partial v_{e}}$, we utilize the adjoint approach, whereby we manipulate (16) and (23) to obtain

$\boldsymbol{\phi}_{j}^{T}\left(\mathbf{K}_{f f}-\omega_{j}^{2} \mathbf{M}_{f f}\right) \boldsymbol{\phi}_{j}-\boldsymbol{\lambda}_{j}^{T} \mathbf{F}_{i n t, f}=\mathbf{0}, \quad j=1, \ldots, n_{c}$,

where as in Eq. $31 \boldsymbol{\lambda}_{j}$ is the adjoint vector and $\mathbf{F}_{i n t, f}=\mathbf{0}$.

Differentiation of Eq. 37 with respect to $v_{e}$ and some manipulations yield

$$
\begin{aligned}
\frac{\partial \omega_{j}^{2}}{\partial v_{e}}= & \boldsymbol{\phi}_{j}^{T}\left(\frac{\partial \mathbf{K}_{f f}}{\partial v_{e}}-\omega_{j}^{2} \frac{\partial \mathbf{M}_{f f}}{\partial v_{e}}\right) \boldsymbol{\phi}_{j}+ \\
& +\left(\frac{\partial\left(\hat{\boldsymbol{\phi}}_{j}^{T} \mathbf{K}_{f f} \hat{\boldsymbol{\phi}}_{j}\right)}{\partial \mathbf{a}_{f}}-\boldsymbol{\lambda}_{j}^{T} \mathbf{K}_{f f}\right) \frac{\partial \mathbf{a}_{f}}{\partial v_{e}}-\boldsymbol{\lambda}_{j}^{T} \frac{\partial \mathbf{F}_{i n t, f}}{\partial v_{e}},
\end{aligned}
$$

where the mass orthonormalization (24) was utilized and the $(\hat{.})$ notation indicates quantities to be treated as constant in the differentiation. To annihilate the implicit sensitivities $\frac{\partial \mathbf{a}_{f}}{\partial v_{e}}$ from Eq. 38, the adjoint vector $\boldsymbol{\lambda}_{j}$ is chosen as the solution to

$\mathbf{K}_{f f} \boldsymbol{\lambda}_{j}=\left(\frac{\partial\left(\hat{\boldsymbol{\phi}}_{j}^{T} \mathbf{K}_{f f} \hat{\boldsymbol{\phi}}_{j}\right)}{\partial \mathbf{a}_{f}}\right)^{T}$,

where the right hand side has been computed by Wallin et al. (2018). Insertion of $\boldsymbol{\lambda}_{j}$ obtained from Eq. 39 into 38 yields 
the sensitivity of the simple eigenvalue $\omega_{j}^{2}$ with respect to each element volume fraction $v_{e}$ as

$$
\frac{\partial \omega_{j}^{2}}{\partial v_{e}}=\boldsymbol{\phi}_{j}^{T}\left(\frac{\partial \mathbf{K}_{f f}}{\partial v_{e}}-\omega_{j}^{2} \frac{\partial \mathbf{M}_{f f}}{\partial v_{e}}\right) \boldsymbol{\phi}_{j}-\lambda_{j}^{T} \frac{\partial \mathbf{F}_{i n t, f}}{\partial v_{e}}
$$

\subsection{Sensitivity analysis of degenerate eigenvalues}

Frequency constrained topology optimization invariably produces designs with repeated, i.e. degenerate, eigenfrequencies. Indeed, $N$-fold degenerate eigenfrequencies of the initial design often exist due to domain symmetry of the body. Additionally, degenerate eigenfrequencies arise during the optimization as multiple simple frequencies $\omega_{j}$ that violate the constraint approach the limiting value $\omega_{\min }$. In either case, the subsequent sensitivity analysis is affected since the eigenmodes corresponding to the $N$-fold degenerate eigenfrequency are not unique, i.e. any set of vectors on the $\mathrm{N}$-dimensioned hyperplane will satisfy the original eigenvalue problem (23), and consequently degenerate eigenfrequencies are not differentiable in the common (Fréchet) sense.

The phenomena of degenerate eigenfrequencies in structural optimization have previously been studied in detail by e.g. Haftka and Gürdal (1992), using the concept of directional derivatives. Later, Seyranian et al. (1994) calculated the directional sensitivities of degenerate eigenfrequencies using a mathematical perturbation method. This formulation has thereafter been implemented in topology optimization by e.g. Du and Olhoff (2007) and Pedersen and Nielsen (2003). The mathematical perturbation-based sensitivity analysis by Seyranian et al. (1994) is limited to linear elasticity and extended here to incorporate nonlinear hyperelasticity.

By definition, the $N$-fold degenerate eigenvalue satisfies

$\bar{\omega}^{2}=\omega_{j}^{2}, \quad j=1, . ., N$,

and its corresponding $\mathbf{M}$-orthonormalized eigenmodes $\boldsymbol{\phi}$ span an $N$-dimensional hyperplane. To begin the sensitivity analysis, we express an eigenmode $\bar{\phi}$ in this hyperplane as

$\bar{\phi}=\sum_{j=1}^{N} \beta_{j} \phi_{j}$.

The choice $\beta_{j}$ of Eq. 42 is made to obtain eigenmodes that remain continuous with respect to design changes in the subsequent sensitivity analysis, cf. Courant and Hilbert (1953).

As done above, we invoke the adjoint method to eliminate the implicit displacement derivatives $\frac{\partial \mathbf{a}}{\partial v_{e}}$. The augmented version of the original eigenvalue problem (23) for the degenerate case is cast as

$\overline{\boldsymbol{\phi}}^{T}\left(\mathbf{K}_{f f}-\bar{\omega}^{2} \mathbf{M}_{f f}\right) \overline{\boldsymbol{\phi}}-\bar{\lambda}^{T} \mathbf{F}_{i n t, f}=0$
; however, the adjoint vector $\bar{\lambda}$ is now ornamented with a $(\bar{\cdot})$ to emphasize the degeneracy, cf. the notation in Eq. 41. Next, we consider the variation of Eq. 43 which yields

$$
\begin{aligned}
& \overline{\boldsymbol{\phi}}^{T}\left(\delta \mathbf{K}_{f f}-\delta \bar{\omega}^{2} \mathbf{M}_{f f}-\bar{\omega}^{2} \delta \mathbf{M}_{f f}\right) \overline{\boldsymbol{\phi}}-\bar{\lambda}^{T} \delta \mathbf{F}_{i n t, f} \\
& +\frac{\partial\left(\hat{\bar{\phi}}^{T} \mathbf{K}_{f f} \hat{\overline{\boldsymbol{\phi}}}\right)}{\partial \mathbf{a}_{f}} \delta \mathbf{a}_{f}-\bar{\lambda}^{T} \frac{\partial \mathbf{F}_{i n t, f}}{\partial \mathbf{a}_{f}} \delta \mathbf{a}_{f}=0,
\end{aligned}
$$

upon utilizing (23). Rearranging the above (44) results in

$$
\begin{aligned}
& \overline{\boldsymbol{\phi}}^{T}\left(\delta \mathbf{K}_{f f}-\bar{\omega}^{2} \delta \mathbf{M}_{f f}\right) \overline{\boldsymbol{\phi}}-\delta \overline{\boldsymbol{\omega}}^{2} \overline{\boldsymbol{\phi}}^{T} \mathbf{M}_{f f} \overline{\boldsymbol{\phi}}-\bar{\lambda}^{T} \delta \mathbf{F}_{i n t, f} \\
& +\left(\frac{\partial\left(\hat{\overline{\boldsymbol{\phi}}}^{T} \mathbf{K}_{f f} \hat{\overline{\boldsymbol{\phi}}}\right)}{\partial \mathbf{a}_{f}}-\bar{\lambda}^{T} \mathbf{K}_{f f}\right) \delta \mathbf{a}_{f}=0 .
\end{aligned}
$$

To annihilate the implicit sensitivities, we require that

$$
\begin{aligned}
\mathbf{K}_{f f} \overline{\boldsymbol{\lambda}} & =\left(\frac{\partial\left(\hat{\overline{\boldsymbol{\phi}}}^{T} \mathbf{K}_{f f} \hat{\overline{\boldsymbol{\phi}}}\right)}{\partial \mathbf{a}_{f}}\right)^{T} \\
& =\sum_{j=1}^{N} \sum_{k=1}^{N} \beta_{j} \beta_{k}\left(\frac{\partial\left(\hat{\boldsymbol{\phi}}_{j}^{T} \mathbf{K}_{f f} \hat{\boldsymbol{\phi}}_{k}\right)}{\partial \mathbf{a}_{f}}\right)^{T},
\end{aligned}
$$

is fulfilled. In Eq. 46, the definition of the $N$-fold eigenmode $\bar{\phi}$ cf. Eq. 42 , is used and in a similar fashion, we now express the adjoint vector $\bar{\lambda}$ as a function of the to-be-determined vectors $\lambda_{j k}$, i.e.

$\bar{\lambda}=\sum_{j=1}^{N} \sum_{k=1}^{N} \beta_{j} \beta_{k} \boldsymbol{\lambda}_{j k}$

which allows (46) to be expressed as

$\sum_{j=1}^{N} \sum_{k=1}^{N} \beta_{j} \beta_{k} \mathbf{K}_{f f} \boldsymbol{\lambda}_{j k}=\sum_{j=1}^{N} \sum_{k=1}^{N} \beta_{j} \beta_{k}\left(\frac{\partial\left(\hat{\boldsymbol{\phi}}_{j}^{T} \mathbf{K}_{f f} \hat{\boldsymbol{\phi}}_{k}\right)}{\partial \mathbf{a}_{f}}\right)^{T}$.

Thus, the implicit sensitivity in Eq. 45 is annihilated for any choice of $\beta_{j}$ and $\beta_{k}, j, k=1, \ldots, N$ if the $\boldsymbol{\lambda}_{j k}$ satisfy

$\mathbf{K}_{f f} \boldsymbol{\lambda}_{j k}=\left(\frac{\partial\left(\hat{\boldsymbol{\phi}}_{j}^{T} \mathbf{K}_{f f} \hat{\boldsymbol{\phi}}_{k}\right)}{\partial \mathbf{a}_{f}}\right)^{T}$.

We solve these $j, k=1, \ldots, N$ adjoint equations for $\boldsymbol{\lambda}_{j k}$, $j, k=1, \ldots, N$. So in all we have to solve $N \times N$ adjoint problems, but due to the symmetry of the right-hand side of Eq. 49 , we note that $\boldsymbol{\lambda}_{j k}=\boldsymbol{\lambda}_{k j}$ and hence we only solve $N(N+1) / 2$ adjoint problems. Also notable is that the computational cost for solving the $N(N+1) / 2$ adjoint 
problems is negligible when using direct solvers since $\mathbf{K}_{f f}$ is identical in all $k, j$-combinations.

Upon inserting (49) into (45), we obtain

$$
\overline{\boldsymbol{\phi}}^{T}\left(\delta \mathbf{K}_{f f}-\bar{\omega}^{2} \delta \mathbf{M}_{f f}\right) \overline{\boldsymbol{\phi}}-\delta \bar{\omega}^{2} \overline{\boldsymbol{\phi}}^{T} \mathbf{M}_{f f} \overline{\boldsymbol{\phi}}-\overline{\boldsymbol{\lambda}}^{T} \delta \mathbf{F}_{i n t, f}=0 .
$$

Use of Eqs. 24, 42, and 47 in Eq. 50 subsequently yields

$$
\begin{gathered}
\sum_{j=1}^{N} \sum_{k=1}^{N} \beta_{j}\left[\phi_{j}^{T}\left(\delta \mathbf{K}_{f f}-\bar{\omega}^{2} \delta \mathbf{M}_{f f}\right) \boldsymbol{\phi}_{k}-\lambda_{j k}^{T} \delta \mathbf{F}_{i n t, f}+\right. \\
\left.-\delta \bar{\omega}^{2} \delta_{j k}\right] \beta_{k}=0 .
\end{gathered}
$$

The above can be arranged as

$\boldsymbol{\beta}^{T}\left[\mathbf{S}-\delta \bar{\omega}^{2} \mathbf{1}\right] \boldsymbol{\beta}=0$,

where $\boldsymbol{\beta}=\left[\beta_{1}, \beta_{2}, \ldots, \beta_{N}\right]^{T}$ and the components of the $N \times N$ matrix $\mathbf{S}$ are

$\mathrm{S}_{j k}=\boldsymbol{\phi}_{j}^{T}\left(\delta \mathbf{K}_{f f}-\bar{\omega}^{2} \delta \mathbf{M}_{f f}\right) \boldsymbol{\phi}_{k}-\boldsymbol{\lambda}_{j k}^{T} \delta \mathbf{F}_{i n t, f}$.

Due to symmetries $\mathbf{K}^{T}=\mathbf{K}, \mathbf{M}^{T}=\mathbf{M}$ and $\boldsymbol{\lambda}_{j k}=\boldsymbol{\lambda}_{k j}$, we see that $\mathbf{S}$ is real and symmetric. To satisfy (52), the matrix $\mathbf{S}-\delta \bar{\omega}^{2} \mathbf{1}$ must be rank-deficient, i.e.

$\operatorname{det}\left[\mathbf{S}-\delta \bar{\omega}^{2} \mathbf{1}\right]=0$,

and thus we see that the eigenvalues of $\mathbf{S}$ provide the directional sensitivities $\delta \bar{\omega}_{j}^{2}, j=1, \ldots, N$ of the degenerate eigenvalue $\bar{\omega}^{2}$.

The directional derivatives $\delta \bar{\omega}_{j}^{2}, j=1, \ldots, N$ of the degenerate eigenvalues are nonlinear functions of the design perturbations $\delta \mathbf{M}_{f f}, \delta \mathbf{K}_{f f}$, and $\delta \mathbf{F}_{i n t, f}$ and do not admit a usual linearization in contrast to simple eigenvalues. However, if $\mathbf{S}$ is diagonal, the directional derivative $\delta \bar{\omega}_{j}^{2}$ can be computed using the simple eigenvalue (37).

Standard gradient-based methods for optimization are not directly applicable for the solution of optimization problems in which degenerate eigenvalues are present due to the lack of differentiability. To combat this, we follow

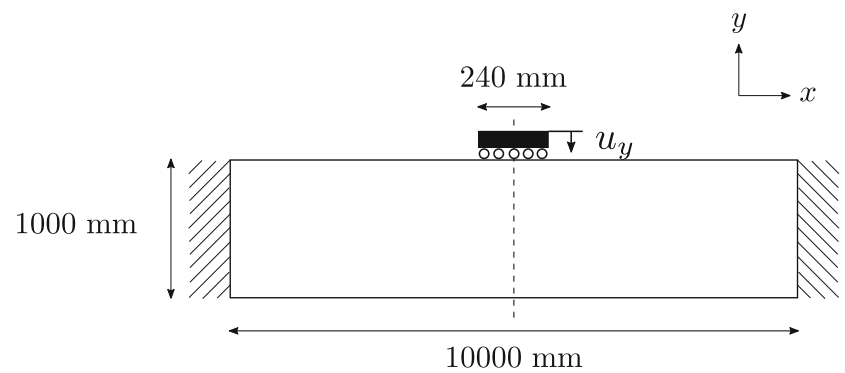

Fig. 1 Double clamped beam. The prescribed displacement $u_{y}$ is uniformly applied to 14 nodes on the center-top surface in the negative $y$-direction
Krog and Olhoff (1999) and Lund (1994), and require S to be diagonal by imposing the constraints

$g_{j k}=\mathrm{S}_{j k}=0, \quad j \neq k, j, k=1, \ldots, N$,

on our optimization problem. In this way, we can always use Eq. 40 to evaluate the derivatives $\frac{\partial \omega_{j}^{2}}{\partial v_{e}}$ of simple eigenvalues and directional derivatives $\delta \bar{\omega}_{j}^{2}, j=1, \ldots, N$ of degenerate eigenvalues. In the MMA, this constraint is easily accommodated in the subproblem wherein $\Delta \mathbf{M}, \Delta \mathbf{K}$, $\Delta \mathbf{r}$ and hence $\mathbf{S}$ are linear in the update $\Delta \mathbf{z}$. As such the $g_{j k}$ sensitivities are trivially evaluated. The final optimization problem $\mathcal{P}$ is thus stated as

$\mathcal{P}\left\{\begin{array}{l}\min _{\mathbf{z}}-g_{o} \\ \text { s.t }\left\{\begin{array}{l}g_{j} \leq 0, \quad j=1, \ldots, n_{c} \\ g_{j k}=0, \quad j \neq k, j, k=1, \ldots, N \\ g_{V} \leq 0 \\ 0 \leq \mathbf{z}_{e} \leq 1, \quad e=1, \ldots, n_{e}\end{array}\right.\end{array}\right.$

\section{Numerical examples}

To illustrate the influence of the eigenfrequency constraints, we design 2D beam-like structures. To connect with previous related work, the material parameters are based on

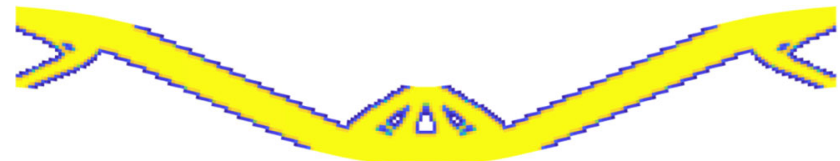

(a)

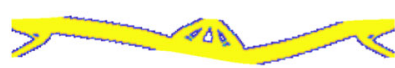

(b) $\boldsymbol{\phi}_{1}$ (c) $\phi_{2}$

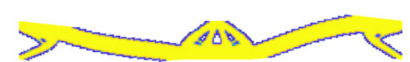

(d) $\phi_{3}$

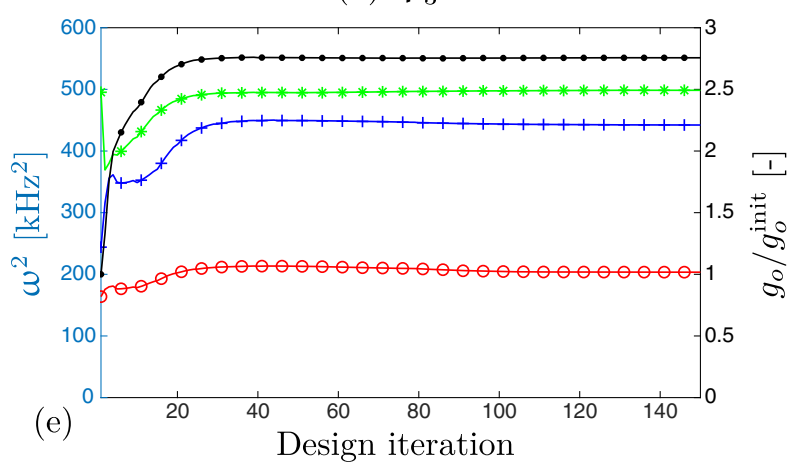

Fig. 2 Optimized design a, first three eigenmodes $\mathbf{b}-\mathbf{d}$, and evolutions of the eigenvalues and compliance ratio $\mathbf{e}$ for the geometry in Fig. 1 when $u_{y}=1000 \mathrm{~mm}$ without eigenvalue constraints. Scaled b$\phi_{1}, \mathbf{c}-\phi_{2}$, and $\mathbf{d}-\phi_{3}$ are plotted for $u_{y}=0 \mathrm{~mm}$. Every 5th iteration is marked in $\mathbf{e}$, where red $-\omega_{1}^{2}$, blue $-\omega_{2}^{2}$, green $-\omega_{3}^{2}$, and black- $g_{o} / g_{o}^{\text {init }}$ 


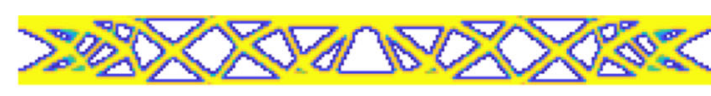

$u_{y} \approx 0[\mathrm{~mm}]$

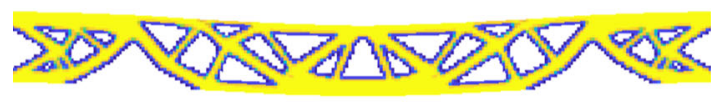

$u_{y}=200[\mathrm{~mm}]$

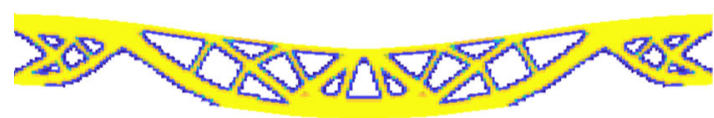

$u_{y}=500[\mathrm{~mm}]$

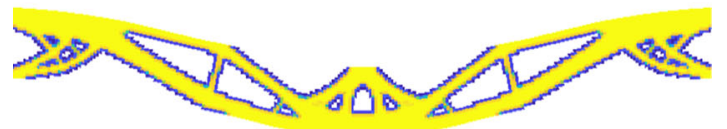

$u_{y}=850[\mathrm{~mm}]$

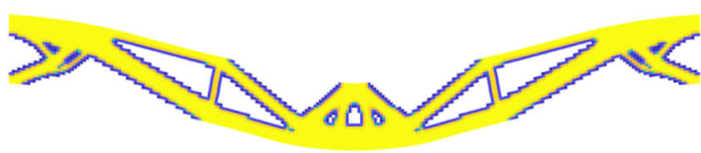

$u_{y}=1000[\mathrm{~mm}]$

Fig. 3 Optimized designs and evolutions of the three smallest eigenvalues and compliance ratio for the geometry in Fig. 1 subject to the eigenvalue constraints $\omega_{j}^{2}>\omega_{\min }^{2}=250 \mathrm{kHz}^{2}$. a $u_{y} \approx 0 \mathrm{~mm}, \mathbf{b}$

the choices by Du and Olhoff (2007), i.e. density $\rho_{g}=$ $1.000 \mathrm{~kg} / \mathrm{m}^{3}$, Young's modulus $E=10.00 \mathrm{MPa}$, and Poisson's ratio $v=0.300$ which provides the bulk and (a)

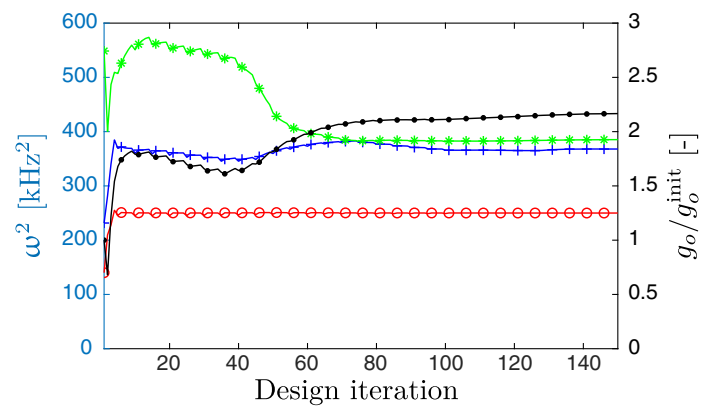

(b)

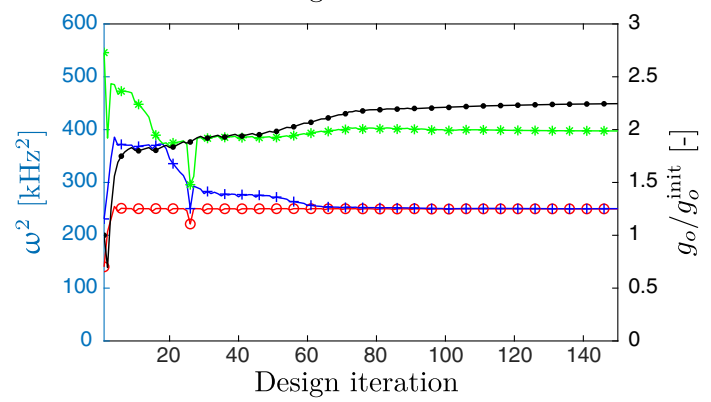

(c)

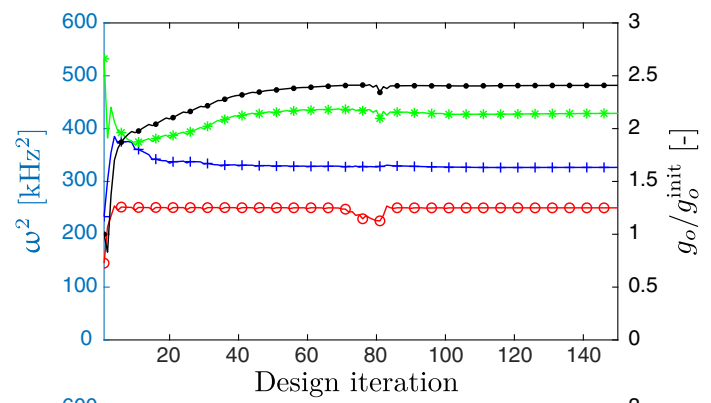

d)

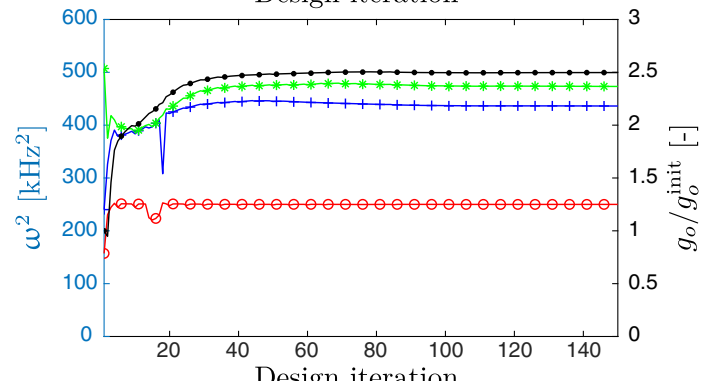

(e)

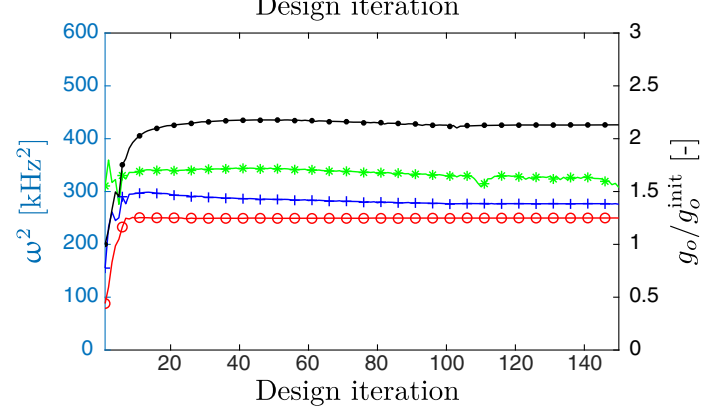

$u_{y}=200 \mathrm{~mm}, \mathbf{c} u_{y}=500 \mathrm{~mm}, \mathbf{d} u_{y}=850 \mathrm{~mm}$, and $\mathbf{e} u_{y}=1000$ $\mathrm{mm}$. In the right hand side plots, every 5 th iteration is marked, where red $-\omega_{1}^{2}$, blue $-\omega_{2}^{2}$, green $-\omega_{3}^{2}$, and black $-g_{o} / g_{o}^{\text {init }}$

shear moduli $K=\frac{E}{3(1-2 v)}$ and $G=\frac{E}{2(1+v)}$. A continuation scheme is utilized for the penalty factor $p, \mathrm{cf}$. Eq. 10, where the initial value $p=2$ is increased by 0.05 every fifth 
Fig. 4 Optimized Fig. 3 a and e designs in their undeformed configurations

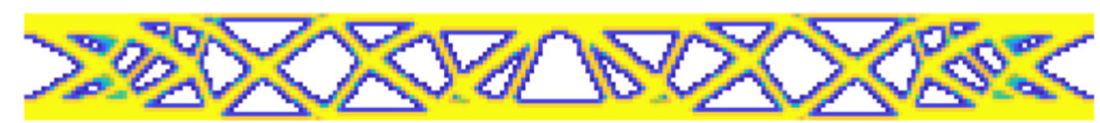

(a)

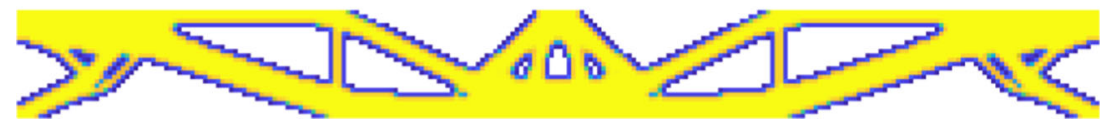

(b) design iteration up to the maximum value $p=3$. The maximum allowed volume is set to $V_{\max }=0.5 \mathrm{~V}$ where $\mathrm{V}$ denotes the volume of the design domain in the reference configuration $\Omega_{o}$. The initial volume fraction distribution is uniform such that $\mathrm{v}_{e}=0.5, e=1, \ldots, n_{e}$. The threshold $\varepsilon_{r}$ for determining when to remove an element is set to $\varepsilon_{r}=$ 0.01 , i.e. the same choice as Bruns and Tortorelli (2003). The numerical parameters used in the MMA algorithm are those proposed by Svanberg (1987).

Two different boundary conditions are considered, namely a double clamped beam and a simply supported beam. The length, width, and out-of-plane thickness of the beam are $10000 \mathrm{~mm}, 1000 \mathrm{~mm}$, and $100 \mathrm{~mm}$, respectively. The beam is discretized using $250 \times 25 \times 1=6250$ eight node fully integrated 3D brick elements, i.e. one element in thickness. Plane strain is assumed and thus the out-of-plane displacement is zero. The filter radius of Eq. 7 is $r=60$ $\mathrm{mm}$, i.e. 1.5 times the element side length.

\subsection{Double clamped beam}

The design domain and boundary conditions for the double clamped 2D beam are shown in Fig. 1 .

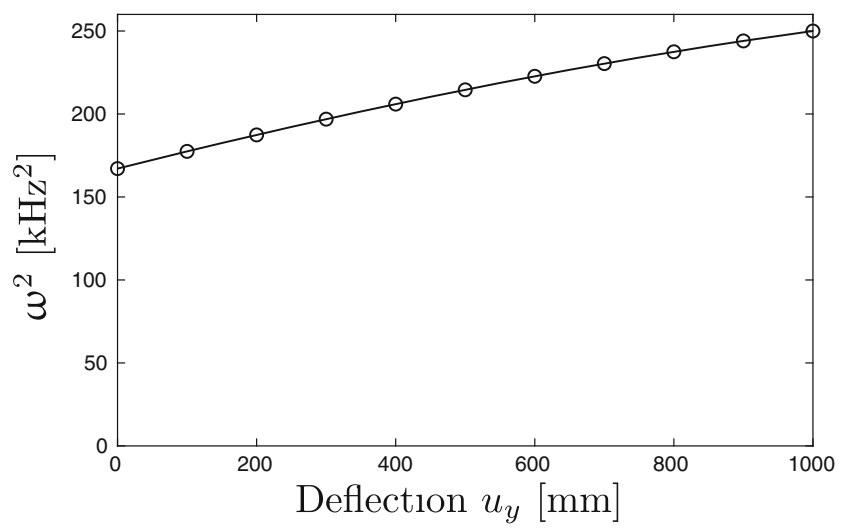

Fig. 5 The smallest eigenvalue as a function of the deflection $u_{z}$ for the Fig. 3e design
As reference, we first investigate the design, structural response, and mode shapes for a $u_{y}=1000 \mathrm{~mm}$ load level when omitting the eigenvalue constraints, with the results
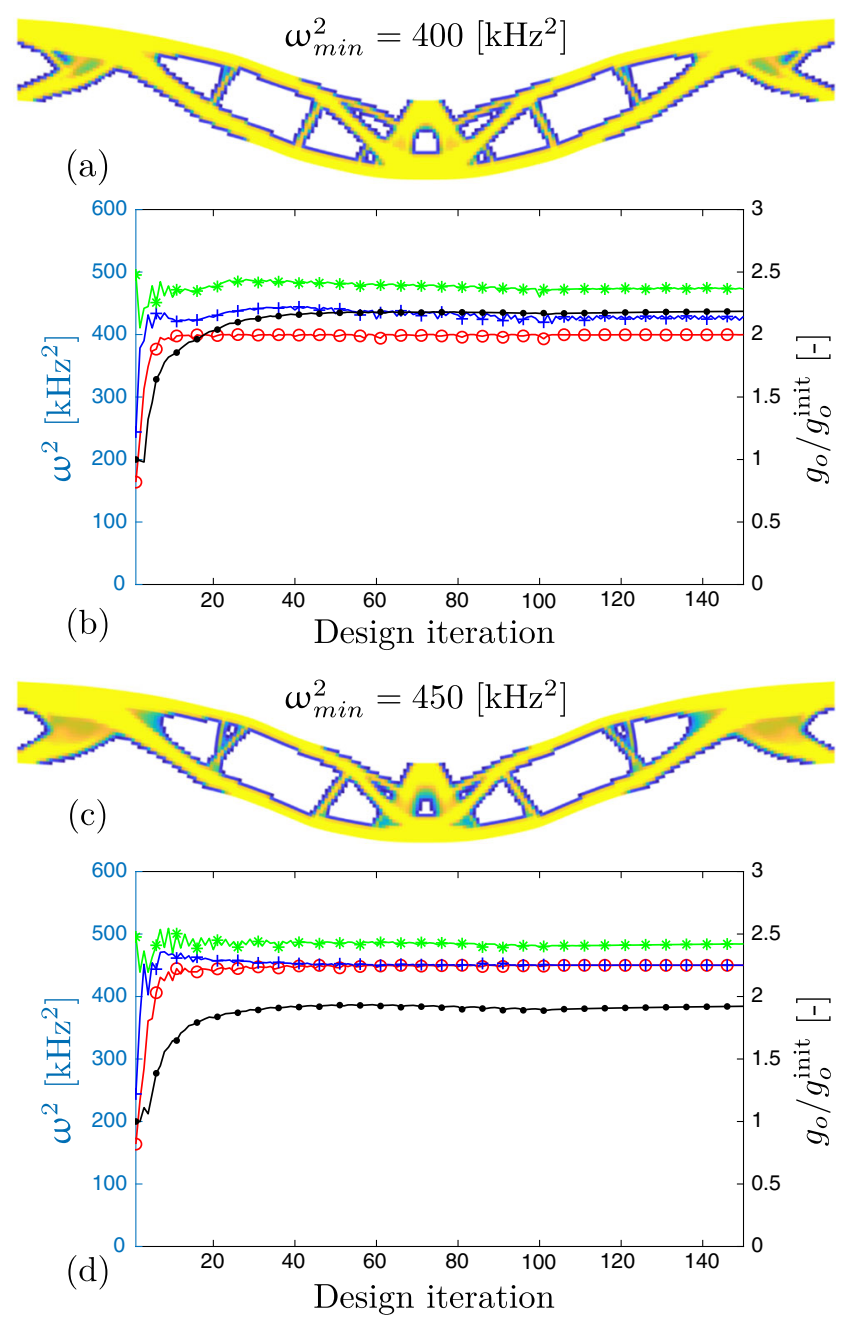

Fig. 6 Optimized designs a, $\mathbf{c}$ and evolutions of the eigenvalues and compliance ratio $\mathbf{b}, \mathbf{d}$ for the geometry in Fig. 1 subject to the eigenvalue constraints $\omega_{j}^{2}>\omega_{\min }^{2}$. a, b $\omega_{\min }^{2}=400 \mathrm{kHz}^{2}$ and $\mathbf{c}, \mathbf{d}$ $\omega_{\min }^{2}=450 \mathrm{kHz}^{2}$. Every 5th iteration is marked in $\mathbf{b}$ and $\mathbf{d}$, where red $-\omega_{1}^{2}$, blue $-\omega_{2}^{2}$, green $-\omega_{3}^{2}$, and black $-g_{o} / g_{o}^{\text {init }}$ 


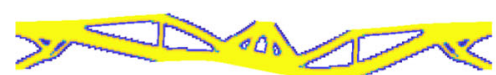

(a) $\phi_{1}$

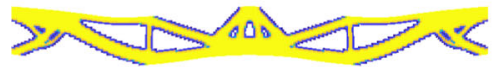

(b) $\boldsymbol{\phi}_{2}$

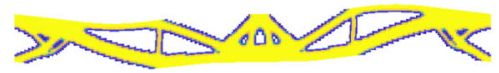

(c) $\phi_{3}$

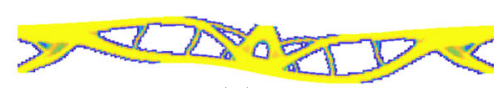

(d) $\phi_{1}$

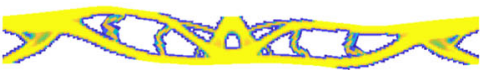

(e) $\phi_{2}$

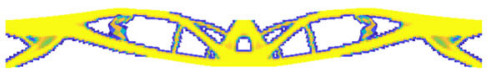

(f) $\phi_{3}$

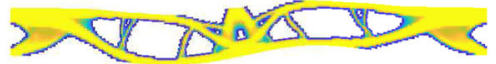

(g) $\phi_{1}$

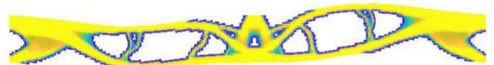

(h) $\boldsymbol{\phi}_{2}$

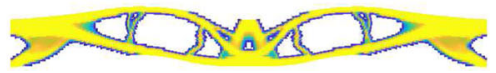

(i) $\phi_{3}$

Fig. 7 Scaled first three eigenmodes of the Figs. $3 \mathrm{e}$ and 6 designs plotted for $u_{y}=0 \mathrm{~mm}$. a-c Fig. 3e design, d-f Fig. $6 \mathrm{a}$ design, and g-i Fig. $6 \mathrm{c}$ design

depicted in Fig. 2. Note that the $\boldsymbol{\phi}_{j}(\boldsymbol{X})=\mathbf{0}, j=1,2,3$ over the $240 \mathrm{~mm}$ top center surface over which the displacement is prescribed.

To investigate the influence of the prestrain in conjunction with the eigenfrequency constraints, we provide examples for five load levels $u_{y}$ with $\omega_{\min }^{2}=250 \mathrm{kHz}^{2}$ fixed. The optimized designs along with the evolutions of the eigenvalues are shown in Fig. 3, where we also plot the compliance ratio $g_{o} / g_{o}^{\text {init }}$.

In Fig. 4, the optimized design corresponding to the $\omega_{j}^{2}>\omega_{\min }^{2}=250 \mathrm{kHz}^{2}$ eigenvalue constraint for the linear $u_{y} \approx 0 \mathrm{~mm}$ and nonlinear $u_{y}=1000 \mathrm{~mm}$ cases is shown in their undeformed configurations. The difference between the designs demonstrates the impact of the prestrain on the structural response. To further illustrate this effect, we compute the smallest eigenvalue of the Fig. 3e design corresponding to different load levels, cf. Fig. 5. As expected, the structure's stiffness increases under load, causing the lowest eigenvalue to increase until it ultimately equals the $250 \mathrm{kHz}^{2}$ constraint limit when $u_{y}=1000$ $\mathrm{mm}$.
Figure 6 illustrates designs subject to minimum eigenvalue constraints $\omega_{\min }^{2}=400 \mathrm{kHz}^{2}$ and $\omega_{\min }^{2}=450 \mathrm{kHz}^{2}$ at the load level $u_{y}=1000 \mathrm{~mm}$. The mode shapes associated with the designs in Figs. 3e and 6 are depicted in Fig. 7. From Figs. 2, 3e, and 6, we conclude that the designs are highly influenced by the eigenvalue constraints.

A comparison of the modal shapes in Figs. 2 and 7 indicates that mode switching occurs during the optimization. Our experience is that this phenomena did not systematically affect the convergence of the optimizer, cf. the evolutions of the eigenvalues and the compliance ratio in Figs. 2, 3, and 6.

In Table 1, the values of $g_{o}, g_{o}^{\text {init }}, \omega_{1}^{2}, \omega_{2}^{2}$, and $\omega_{3}^{2}$ for each design are given. An interesting observation is the absence of degenerate eigenfrequencies. The smallest observed relative difference between two eigenfrequencies during all design iterations is $\approx 10^{-5}$, i.e. the eigenfrequencies are always treated as simple. The last row in Table 1 shows the performance for the Fig. 6a design which is optimized for $u_{y} \approx 0 \mathrm{~mm}$ when it is subjected to the $u_{y}=1000 \mathrm{~mm}$ displacement. A comparison with the performance of the
Table 1 Resulting values for the double clamped beam designs

\begin{tabular}{lllllll}
\hline$\omega_{\text {min }}^{2}\left[\mathrm{kHz}^{2}\right]$ & $u_{y}[\mathrm{~mm}]$ & $\omega_{1}^{2}\left[\mathrm{kHz}^{2}\right]$ & $\omega_{2}^{2}\left[\mathrm{kHz}^{2}\right]$ & $\omega_{3}^{2}\left[\mathrm{kHz}^{2}\right]$ & $g_{o}[\mathrm{kNm}]$ & $g_{o}^{\text {init }}[\mathrm{kNm}]$ \\
\hline 0.000 & 1000 & 203.4 & 442.2 & 498.5 & 16.04 & 5.818 \\
250.0 & 0.000 & 250.0 & 368.0 & 385.3 & $\approx 0.000$ & $\approx 0.000$ \\
250.0 & 200.0 & 250.0 & 250.4 & 397.4 & 0.361 & 0.161 \\
250.0 & 500.0 & 250.0 & 326.6 & 428.9 & 2.675 & 1.110 \\
250.0 & 850.0 & 250.0 & 436.2 & 473.3 & 9.624 & 3.853 \\
250.0 & 1000 & 250.0 & 478.7 & 479.9 & 14.75 & 5.818 \\
400.0 & 1000 & 399.8 & 428.9 & 472.8 & 12.72 & 5.818 \\
450.0 & 1000 & 449.9 & 450.1 & 484.2 & 11.18 & 5.818 \\
250.0 & $(0 \rightarrow) 1000$ & 173.8 & 243.4 & 305.7 & 8.016 & - \\
\hline
\end{tabular}

The volume constraint is active for all examples 

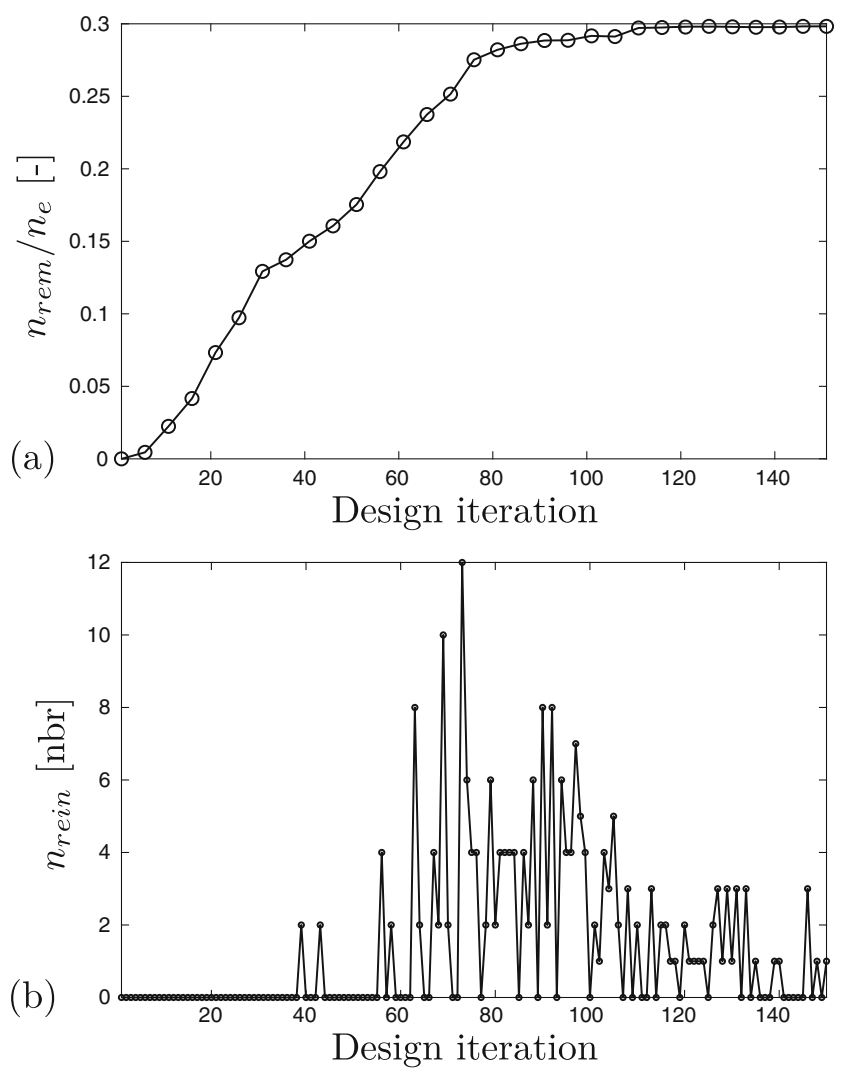

Fig. 8 a Evolution of the ratio of removed finite elements $n_{\text {rem }}$ with respect to the total number of elements. b The number of reintroduced elements $n_{\text {rein }}$ during a design update. Both (a) and (b) correspond to the Fig. 3 b design. Every 5 th iteration is marked in (a)

Fig. 6d design clearly illustrates the impact of the nonlinear framework.

The evolution of the number of removed and reintroduced finite elements is depicted for the Fig. 3b design in Fig. 8. We note that the percentage of removed elements reaches a terminal value of $\approx 30 \%$, and that the number of

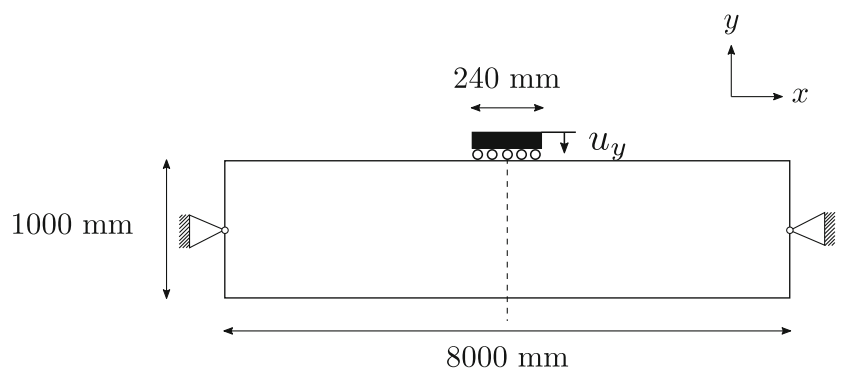

Fig. 9 Simply supported beam. The prescribed displacement $u_{y}$ is uniformly applied to 14 nodes on the center-top surface in the negative $y$-direction elements that are reintroduced during a design update rarely exceeds 10 elements in a 6250 element mesh.

\subsection{Simply supported beam}

In the last example, we consider the simply supported beam shown in Fig. 9.

Again, we initially apply the $u_{y}=1000 \mathrm{~mm}$ load level and omit the eigenvalue constraints which yields the design and mode shapes depicted in Fig. 10. Thereafter, we investigate the influence of the prestrain and use the load $u_{y} \approx 0 \mathrm{~mm}$ to mimic a linear design. Imposing the constraint $\omega_{j}^{2}>\omega_{\min }^{2}=150 \mathrm{kHz}^{2}$ renders the design and its mode shapes illustrated in Fig. 11.

Figure 12 illustrates designs at the $u_{y}=1000 \mathrm{~mm}$ prestrain subject to the eigenvalue constraints $\omega_{j}^{2}>\omega_{\min }^{2}$ with $\omega_{\min }^{2}=150 \mathrm{kHz}^{2}$ and $\omega_{\min }^{2}=250 \mathrm{kHz}^{2}$. Similar to the previous example, we observe great influences of the eigenvalue constraints on the final designs. We again emphasize the lack of degenerate eigenfrequencies.

The mode shapes associated with the Fig. 12 designs are plotted in Fig. 13. We observe that a local eigenmode

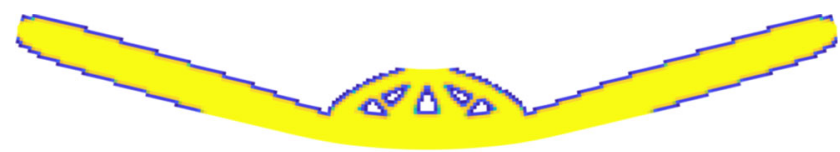

(a)

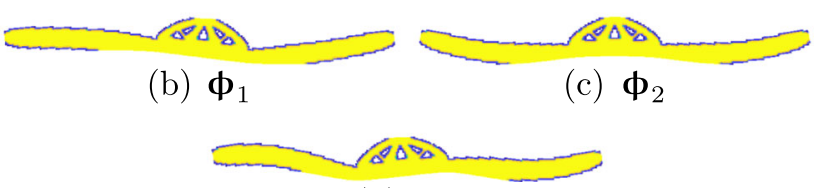

(d) $\phi_{3}$

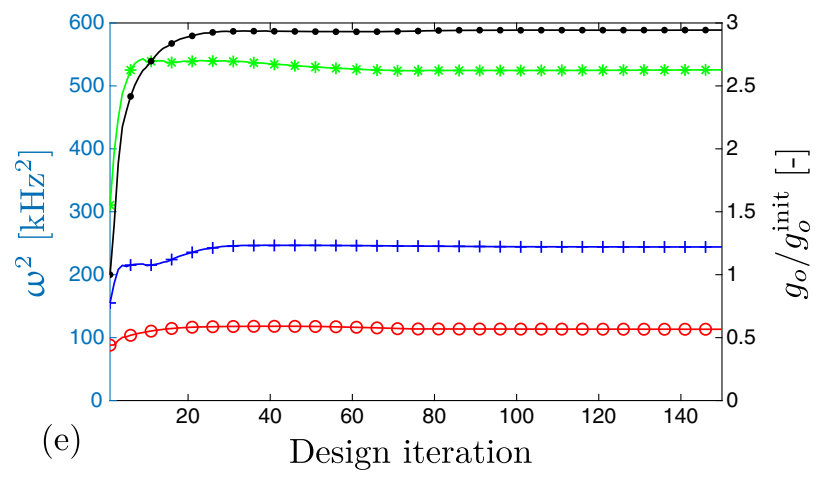

Fig. 10 Optimized design a, evolutions of the eigenvalues and compliance ratio $\mathbf{b}$, and first three eigenmodes $\mathbf{c}-\mathbf{e}$ for the geometry in Fig. 9 when $u_{y}=1000 \mathrm{~mm}$ without eigenvalue constraints. Scaled $\mathbf{c}-\boldsymbol{\phi}_{1}, \mathbf{d}-\boldsymbol{\phi}_{2}$, and $\mathbf{e}-\boldsymbol{\phi}_{3}$ are plotted for $u_{y}=0 \mathrm{~mm}$. Every 5 th iteration is marked in $\mathbf{b}$ where red $-\omega_{1}^{2}$, blue $-\omega_{2}^{2}$, green- $\omega_{3}^{2}$, and black- $g_{o} / g_{o}^{\text {init }}$ 


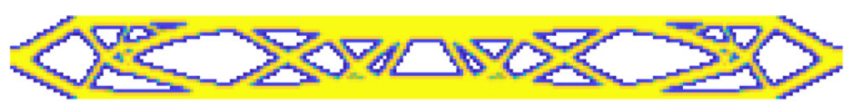

(a)

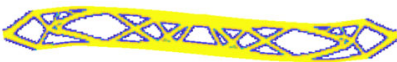

(c) $\phi_{1}$

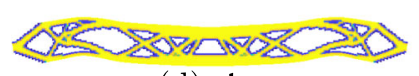

(d) $\phi_{2}$

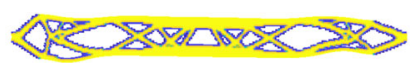

(e) $\phi_{3}$

Fig. 11 Optimized design a, evolutions of the eigenvalues and compliance ratio $\mathbf{b}$, and first three eigenmodes $\mathbf{c}-\mathbf{e}$ of a simply supported beam when $u_{y} \approx 0 \mathrm{~mm}$ subject to the eigenvalue constraints $\omega_{j}^{2}>$

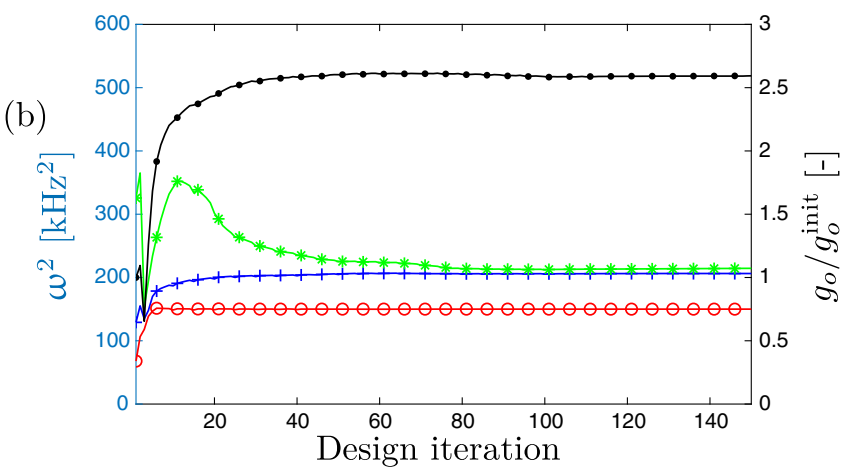

$\omega_{\min }^{2}=150 \mathrm{kHz}^{2}$. Scaled $\mathbf{c}-\boldsymbol{\phi}_{1}, \mathbf{d}-\boldsymbol{\phi}_{2}$, and $\mathbf{e}-\boldsymbol{\phi}_{3}$ are plotted for $u_{y}=0 \mathrm{~mm}$. Every 5 th iteration is marked in $(\mathbf{b})$ where red $-\omega_{1}^{2}$, blue $-\omega_{2}^{2}$, and green- $\omega_{3}^{2}$
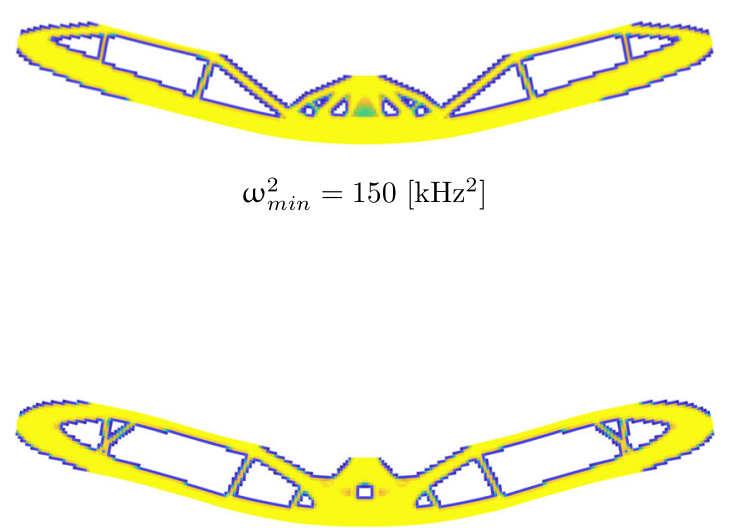

$\omega_{\min }^{2}=250\left[\mathrm{kHz}^{2}\right]$

Fig. 12 Optimized designs and evolutions of the eigenvalues and compliance ratio for the geometry in Fig. 9 subject to the eigenvalue constraints $\omega_{j}^{2}>\omega_{\min }^{2} \cdot \mathbf{a} \omega_{\min }^{2}=150 \mathrm{kHz}^{2}$ and $\mathbf{b} \omega_{\min }^{2}=250$ (a)

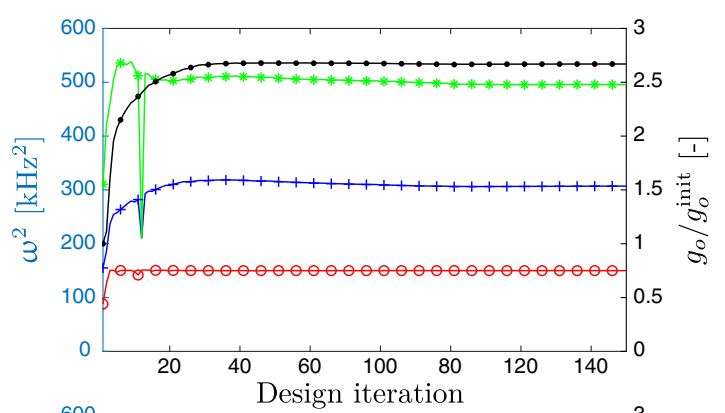

(b)

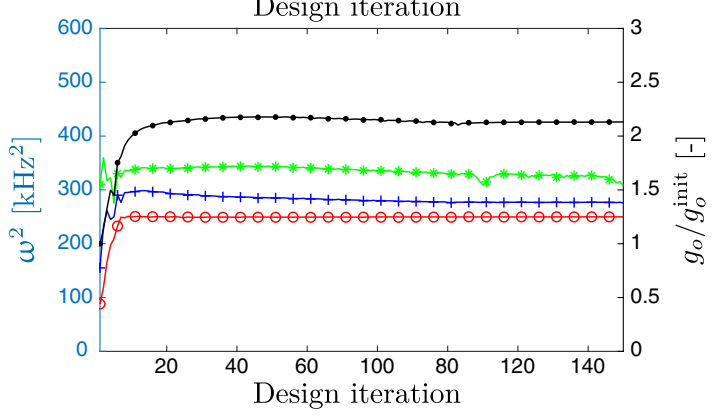

$\mathrm{kHz}^{2}$. In the right hand side plots, every 5 th iteration is marked, where red $-\omega_{1}^{2}$, blue $-\omega_{2}^{2}$, green- $\omega_{3}^{2}$, and black $-g_{o} / g_{o}^{\text {init }}$

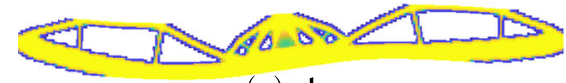

(a) $\phi_{1}$

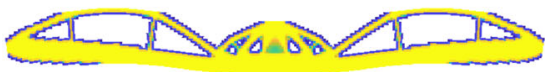

(b) $\boldsymbol{\phi}_{2}$

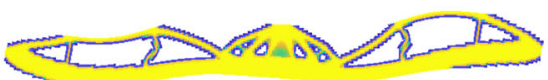

(c) $\boldsymbol{\phi}_{3}$

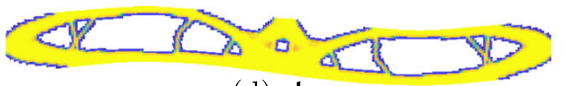

(d) $\boldsymbol{\phi}_{1}$

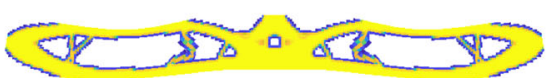

(e) $\boldsymbol{\phi}_{2}$

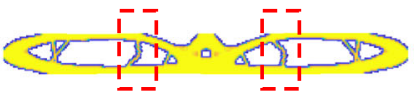

(f) $\phi_{3}$

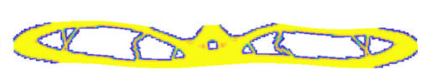

(g) $\boldsymbol{\phi}_{3}$

Fig. 13 First three eigenmodes of the Fig. 12 designs in their undeformed configurations. a-c Fig. 12a and d-g Fig. 12b. f, $\mathbf{g}$ The third eigenmode for two different design iterations. First row $-\phi_{1}$, second row $-\phi_{2}$, and third row $-\phi_{3}$ 
Fig. 14 Optimized Figs. 11 and 12 a designs in their undeformed configurations.

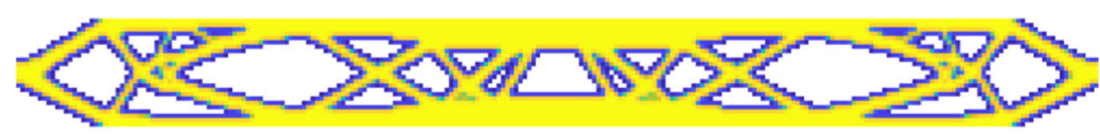

(a)

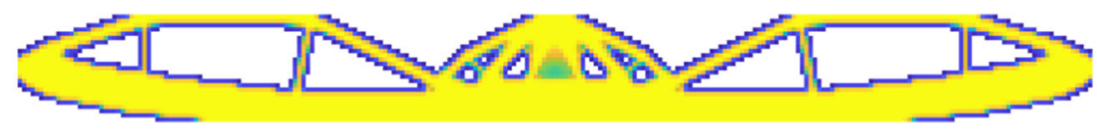

(b)
Table 2 Resulting values in the last design iteration for the simply supported beam

\begin{tabular}{lllllll}
\hline$\omega_{\min }^{2}\left[\mathrm{kHz}^{2}\right]$ & $u_{y}[\mathrm{~mm}]$ & $\omega_{1}^{2}\left[\mathrm{kHz}^{2}\right]$ & $\omega_{2}^{2}\left[\mathrm{kHz}^{2}\right]$ & $\omega_{3}^{2}\left[\mathrm{kHz}^{2}\right]$ & $g_{o}[\mathrm{kNm}]$ & $g_{o}^{\text {init }}[\mathrm{kNm}]$ \\
\hline 0.000 & 1000 & 113.3 & 244.1 & 525.5 & 8.701 & 2.956 \\
150.0 & $\approx 0.000$ & 150.0 & 206.3 & 214.5 & $\approx 0.000$ & $\approx 0.000$ \\
150.0 & 1000 & 150.0 & 307.1 & 495.7 & 7.892 & 2.956 \\
250.0 & 1000 & 249.8 & 275.9 & 307.7 & 6.300 & 2.956 \\
150.0 & $(0 \rightarrow) 1000$ & 135.9 & 212.0 & 216.4 & 4.293 & - \\
\hline
\end{tabular}

The volume constraint is active for all examples highlighted in Fig. 13f has emerged, whereas for a previous design iteration, the global mode displayed in Fig. 13g was found instead. This phenomena can be identified by the small drops in the evolution of the third eigenvalue in Fig. 12.

We compare the optimized designs corresponding to $u_{y} \approx 0 \mathrm{~mm}$ and $u_{y}=1000 \mathrm{~mm}$ subject to the $\omega_{j}^{2}>$ $\omega_{\min }^{2}=150 \mathrm{kHz}^{2}$ eigenvalue constraint in the undeformed configurations in Fig. 14. The impact of the prestrain on the optimized topology is clear.

In Table 2, the values of the response quantities of interest are listed for the various designs. It is concluded that the optimization problem $\mathcal{P}$ succeeds in drastically increasing the fundamental eigenfrequency. Again, we evaluate the impact of the nonlinear framework by analyzing the performance of the Fig. 11 design obtained for $u_{y} \approx 0$ $\mathrm{mm}$ at a $u_{y}=1000 \mathrm{~mm}$ load level, cf. the last row in Table 2. As expected, the Fig. 12a design outperforms its linear counterpart at this load level.

\section{Conclusions}

The mathematical framework for performing topology optimization considering eigenfrequencies is extended to finite strain hyperelastic structures subjected to small harmonic oscillations about a prestrained equilibrium configuration. A stiff structure is obtained, while constraining the lowest eigenfrequencies and the maximum allowable volume. The low volume fraction element removal method proposed by Bruns and Tortorelli (2003) is implemented to mitigate problems associated with nearly void regions in finite deformation eigenfrequency topology optimization, i.e. gross distortion and spurious eigenmodes. The problem in the sensitivity analysis associated with the nondifferentiability of degenerate eigenfrequencies is treated by extending the perturbation method of Seyranian et al. (1994) to include both geometrical and material nonlinearities.

The numerical examples show that the fundamental eigenfrequency of prestrained structures can be increased significantly using the proposed framework. The sensitivity analysis implementation works for both simple and degenerate eigenfrequencies. As expected, the magnitude of the prestrain significantly affects the optimized designs.

Acknowledgments This work was performed under the auspices of the U.S. Department of Energy by Lawrence Livermore Laboratory under Contract DE-AC52-07NA27344. The authors would finally like to thank Prof. Krister Svanberg for providing the MMA code.

Funding information Open access funding provided by Lund University. The financial supports from the Swedish research council (grant nbr. 2015-05134) and the Swedish energy agency (grant. nbr 48344-1) are also gratefully acknowledged.

\section{Compliance with ethical standards}

Conflict of interest The authors declare that they have no conflict of interest.

Replication of results The optimization problem $\mathcal{P}$ is solved using the in-house Fortran 90 implementation summarized in Algorithm 1 together with the MMA code from Prof. Krister Svanberg. Linear equation systems and eigenvalue problems are solved using Intel MKL Pardiso 2018 and FEAST Eigenvalue Solver 2.0, respectively. The geometry and mesh are generated using Abaqus 2019. 


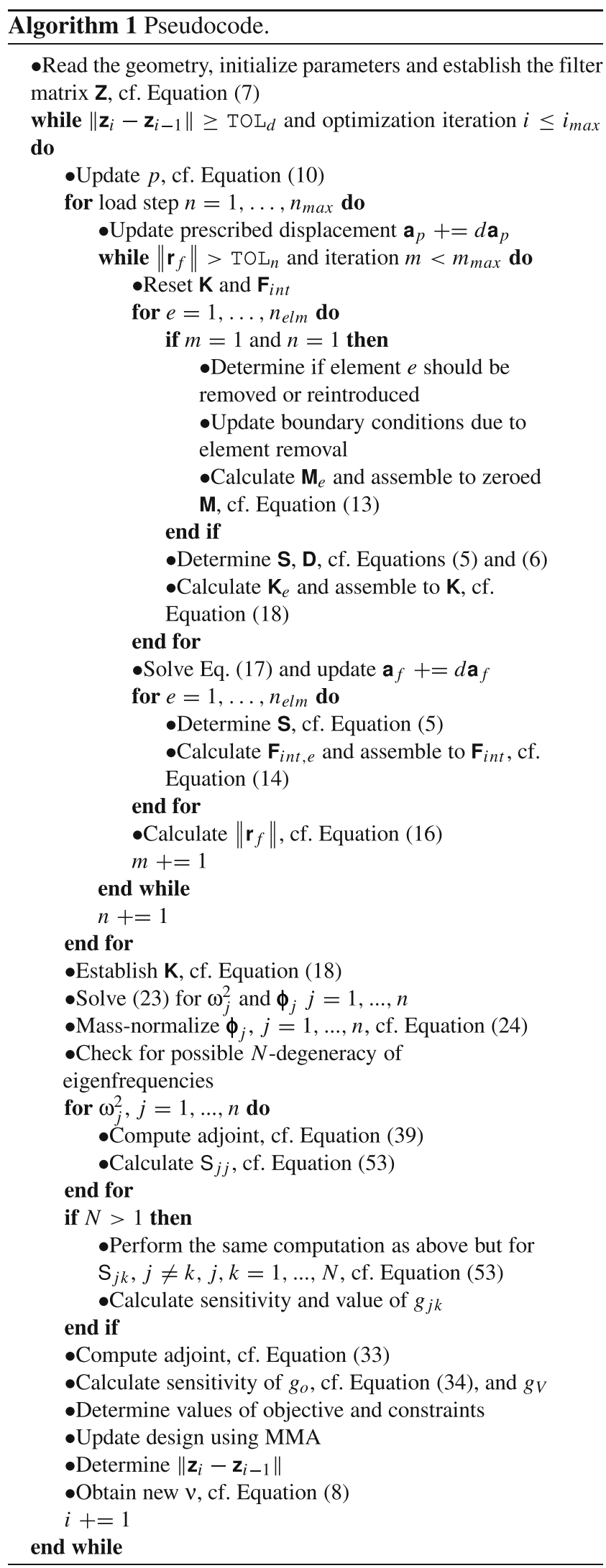

Open Access This article is licensed under a Creative Commons Attribution 4.0 International License, which permits use, sharing, adaptation, distribution and reproduction in any medium or format, as long as you give appropriate credit to the original author(s) and the source, provide a link to the Creative Commons licence, and indicate if changes were made. The images or other third party material in this article are included in the article's Creative Commons licence, unless indicated otherwise in a credit line to the material. If material is not included in the article's Creative Commons licence and your intended use is not permitted by statutory regulation or exceeds the permitted use, you will need to obtain permission directly from the copyright holder. To view a copy of this licence, visit http:// creativecommonshorg/licenses/by/4.0/.

\section{Appendix}

\section{Verification of sensitivities}

The accuracy of the analytical sensitivity expression is verified by numerical differentiation using the forward finite difference method. Due to the finite precision in the eigenfrequency computations, the degeneracy must be determined to within a given tolerance $\varepsilon_{t o l}$, cf. Seyranian et al. (1994). To set this tolerance, we compare the analytical directional derivative obtained when assuming simple or degenerate eigenfrequencies, i.e. $\delta \omega_{j}^{2}$ when using Eq. 40 $\mathrm{D}$ or $\delta \omega_{j}^{2}$ when using Eq. 54, to the forward finite difference derivative approximation

$$
\delta \omega_{j}^{2} \approx \frac{\omega_{j}^{2}\left(\mathrm{z}_{1}, \ldots, \mathrm{z}_{e}+\varepsilon, \ldots, \mathrm{z}_{n_{e}}\right)-\omega_{j}^{2}\left(\mathrm{z}_{1}, \ldots, \mathrm{z}_{n_{e}}\right)}{\varepsilon},
$$

where $\varepsilon$ denotes the perturbation of variable $\mathrm{z}_{e}$. Three different scenarios are then possible. Firstly, if $\delta \omega_{j}^{2} \approx$ $\delta \omega_{j}^{2}$ and $\delta \omega_{j}^{2} \neq \delta \omega_{j}^{2}$, the eigenfrequency is treated as

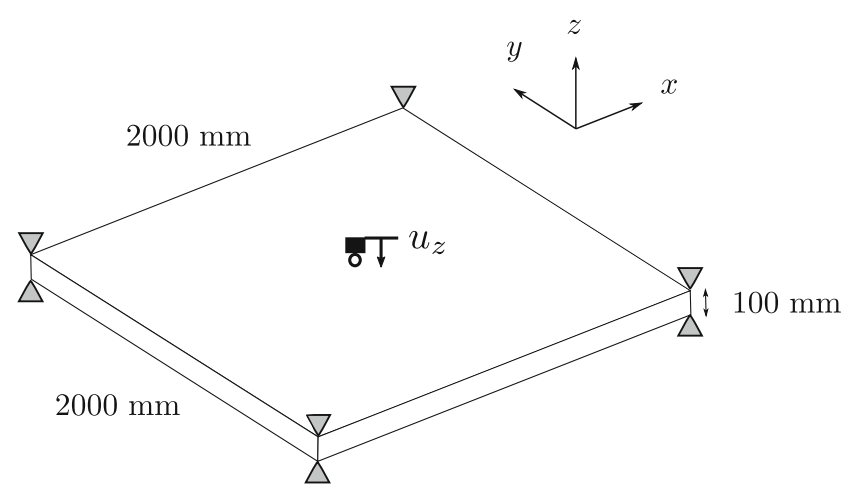

Fig. 15 Simply supported 3D plate. The prescribed displacement $u_{z}$ is applied to the top-center surface in the negative $z$-direction 

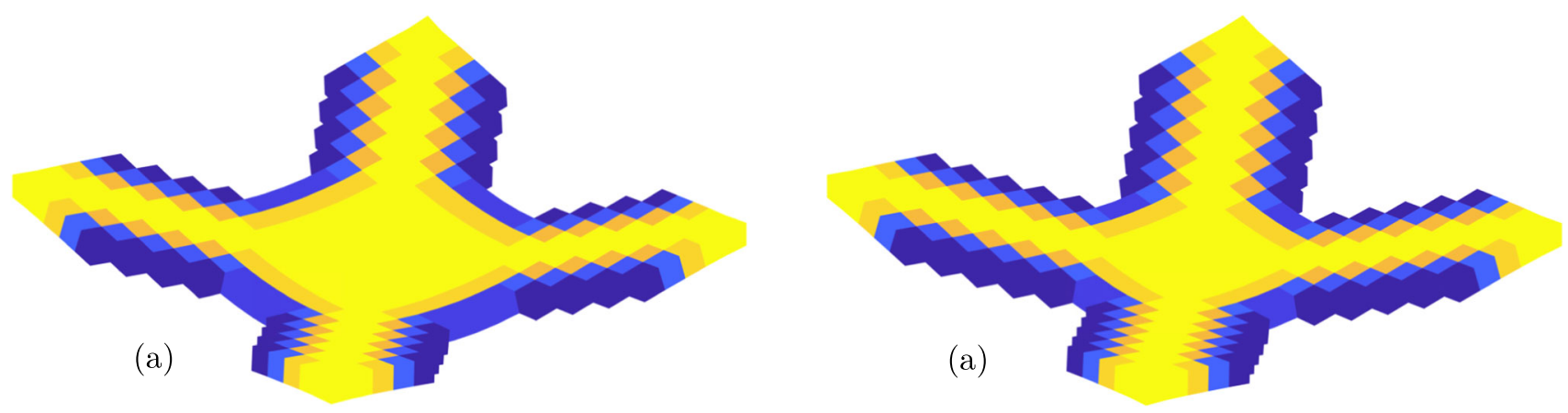

(b) $\phi_{1}$

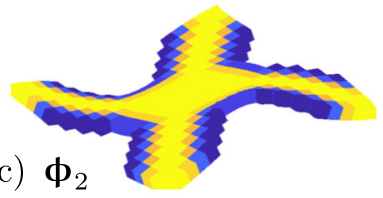

(b) $\boldsymbol{\phi}_{1}$
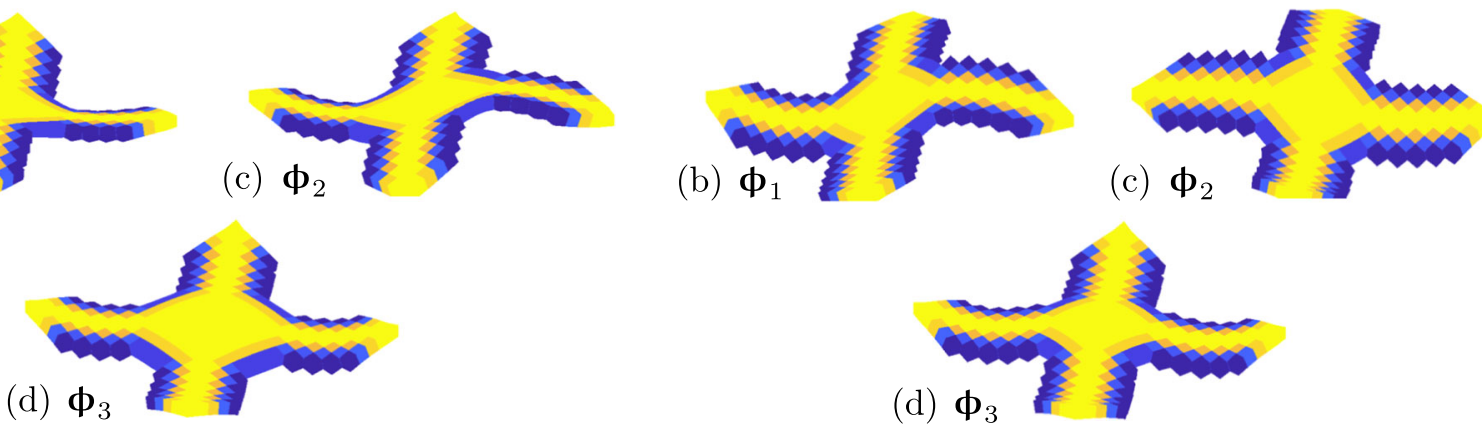

Fig. 16 Optimized design $\mathbf{a}$ and first three eigenmodes $\mathbf{b}-\mathbf{d}$ of simply supported 3D plate when $u_{z}=280 \mathrm{~mm}$ without eigenvalue constraints. Scaled $\mathbf{b}-\phi_{1}, \mathbf{c}-\phi_{2}$, and $\mathbf{d}-\phi_{3}$ are plotted for $u_{z}=0 \mathrm{~mm}$

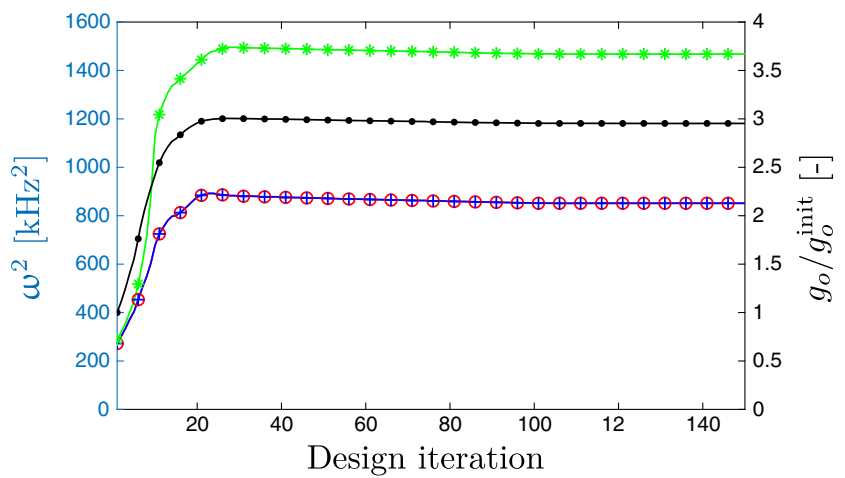

Fig. 17 Evolutions of the eigenvalues and compliance ratio value for the Fig. 16 design. Every 5 th iteration is marked. Red $-\omega_{1}^{2}$, blue- $\omega_{2}^{2}$, green- $\omega_{3}^{2}$, and black- $g_{o} / g_{o}^{\text {init }}$

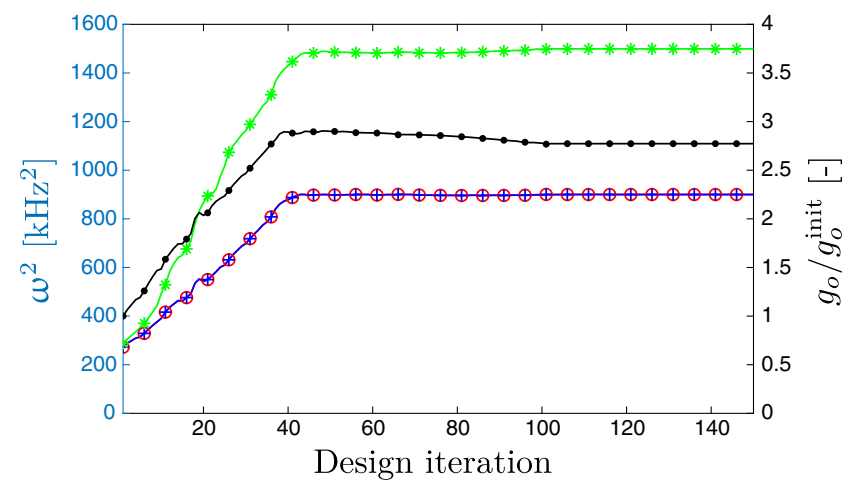

Fig. 19 Evolutions of the eigenvalues and compliance ratio value for the Fig. 18 design. Every 5 th iteration is marked. Red- $\omega_{1}^{2}$, blue- $\omega_{2}^{2}$, green $-\omega_{3}^{2}$, and black $-g_{o} / g_{o}^{\text {init }}$
Table 3 Resulting values for the 3D plate designs

\begin{tabular}{llllll}
\hline$\omega_{\min }^{2}\left[\mathrm{kHz}^{2}\right]$ & $\omega_{1}^{2}\left[\mathrm{kHz}^{2}\right]$ & $\omega_{2}^{2}\left[\mathrm{kHz}^{2}\right]$ & $\omega_{3}^{2}\left[\mathrm{kHz}^{2}\right]$ & $V^{*} / V[-]$ & $g_{o}[\mathrm{kNm}]$ \\
\hline 0.000 & 851.4 & 851.4 & 1468 & 0.500 & 1.653 \\
900.0 & 900.0 & 900.0 & 1499 & 0.460 & 1.563 \\
\hline
\end{tabular}

For both designs $u_{z}=280 \mathrm{~mm}$ and $g_{o}^{\text {init }}=0.560 \mathrm{kNm}$ 
simple. Secondly, if $\delta \omega_{j}^{2} \approx \delta \omega_{j}^{2}$ and $\delta \omega_{j}^{2} \neq \delta \omega_{j}^{2}$, the eigenfrequency is treated as degenerate. Finally, if both $\mathrm{S} \quad \mathrm{D} \quad \mathrm{FD}$

$\delta \omega_{j}^{2} \approx \delta \omega_{j}^{2} \approx \delta \omega_{j}^{2}, \mathbf{S}$ is diagonal and the eigenvalue is degenerate, but Eqs. 40 and 54 yield identical result, cf. Seyranian et al. (1994).

Based on the analysis above, we conclude that $N>1$ eigenvalue is $N$-fold degenerate when they agree to within the tolerance of $\varepsilon_{t o l}=10^{-8}$. This choice is smaller than the tolerance used by e.g. Seyranian et al. (1994). Surely, this is a case-by-case choice. For example, here we are dealing with nonlinear boundary value problem, and thus structural equilibrium is only ensured to within a given tolerance. Another factor affecting the value of $\varepsilon_{t o l}$ is the eigensolver; We use the FEAST Eigenvalue Solver 2.0, cf. Polizzi (2009).

The problem considered for verification of the sensitivity computation is a simply supported 3D plate-like structure cf. the design domain in Fig. 15. The length, width, and thickness of the plate are, $2000 \mathrm{~mm}, 2000 \mathrm{~mm}$, and 100 $\mathrm{mm}$, respectively. We use a coarse mesh that consists of $20 \times 20 \times 1=400$ fully integrated 3D brick elements. The filter radius, cf. Eq. 7, is $r=150 \mathrm{~mm}$, i.e. 1.5 times the element side length.

This geometry is chosen due to its inherent domain symmetry which is ideal for verifying the sensitivities of degenerate eigenfrequencies. First, we obtain an optimized design without constraining the eigenvalue. The resulting design and associated mode shapes appear in Fig. 16, the evolutions of the eigenvalue and the compliance ratio are plotted in Fig. 17, and the compliance and the total volume fraction $\left(\int_{\Omega_{0}} v d v\right) / V=V^{*} / V$ are denoted in Table 3.

Next, we repeat the previous problem, but now we enforce the eigenvalue constraints $\omega_{j}^{2}>\omega_{\min }^{2}=900 \mathrm{kHz}^{2}$. The resulting design is depicted in Fig. 18, where the mode shapes also are seen.

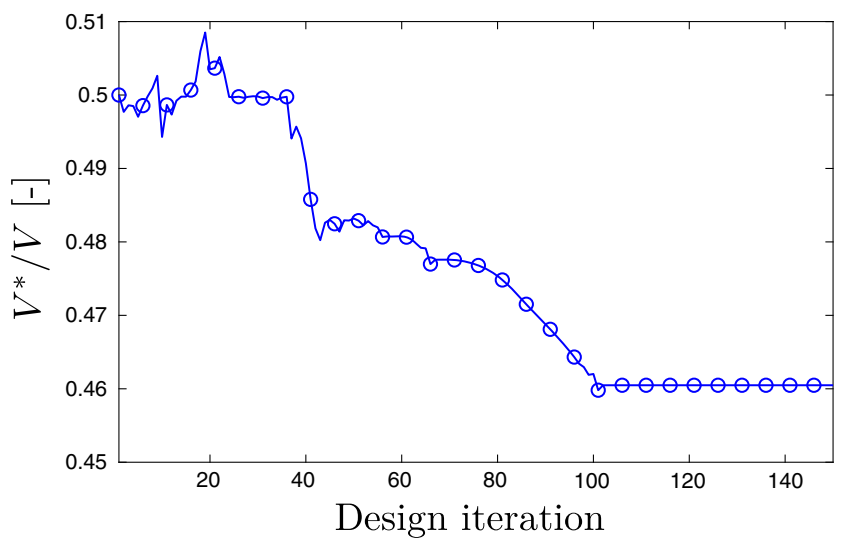

Fig. 20 Evolution of the total volume fraction $V^{*} / V$ for the Fig. 18 design. Every 5 th iteration is marked.
As is shown in Fig. 19, the twofold degenerate fundamental eigenvalue is increased to fulfill the lower bound $\omega_{\min }^{2}=900 \mathrm{kHz}^{2}$ constraint. Since a coarse mesh is used to discretize the domain, the optimization algorithm is working in a relatively small design space, which might explain such small differences between the Figs. 16 and 18 designs. An interesting phenomenon is noted as the Fig. 18 design does not utilize all available material, cf. Fig. 20. Similar observations are made in Stolpe (2010), who discusses the nonnecessity of an active volume constraint at an optimal solution for continuous and discrete design problems. The terminal values of the constrained eigenvalues and $g_{o}$ are denoted in Table 3 .

\section{References}

Allaire G, Jouve F (2005) A level-set method for vibration and multiple loads structural optimization. Comput Methods Appl Mech Eng 194(30-33):3269-3290

Bendsøe MP (1989) Optimal shape design as a material distribution problem. Struct Optim 1(4):193-202

Bourdin B (2001) Filters in topology optimization. Int J Numer Methods Eng 50(9):2143-2158

Bruns TE, Tortorelli DA (2001) Topology optimization of non-linear elastic structures and compliant mechanisms. Comput Methods Appl Mech Eng 190(26-27):3443-3459

Bruns TE, Sigmund O, Tortorelli DA (2002) Numerical methods for the topology optimization of structures that exhibit snap-through. Int J Numer Methods Eng 55(10):1215-1237

Bruns TE, Tortorelli DA (2003) An element removal and reintroduction strategy for the topology optimization of structures and compliant mechanisms. Int J Numer Methods Eng 57(10):14131430

Buhl T, Pedersen CB, Sigmund O (2000) Stiffness design of geometrically nonlinear structures using topology optimization. Struct Multidiscip Optim 19(2):93-104

Clough RW, Penzien J (1993) Dynamics of structures. Singapore, McGraw Hill

Courant R, Hilbert D (1953) Methods of mathematical physics, vol I. Intersci. Publ. Inc., New York, p 106

Crisfield MA (1993) Non-linear finite element analysis of solids and structures, vol 1. Wiley, New York

Díaz AR, Kikuchi N (1992) Solutions to shape and topology eigenvalue optimization problems using a homogenization method. Int J Numer Methods Eng 35(7):1487-1502

Du J, Olhoff N (2007) Topological design of freely vibrating continuum structures for maximum values of simple and multiple eigenfrequencies and frequency gaps. Struct Multidiscip Optim 34(2):91-110

Ferrari F, Lazarov BS, Sigmund O (2018) Eigenvalue topology optimization via efficient multilevel solution of the frequency response. Int J Numer Methods Eng 115(7):872-892

Haftka RT, Gürdal Z (1992) Elements of structural optimization. Kluwer Academic Publishers, Dordrecht

Ivarsson N, Wallin M, Tortorelli D (2018) Topology optimization of finite strain viscoplastic systems under transient loads. Int J Numer Methods Eng 114(13):1351-1367

Kemmler R, Lipka A, Ramm E (2005) Large deformations and stability in topology optimization. Struct Multidiscip Optim 30(6):459-476 
Klarbring A, Strömberg N (2013) Topology optimization of hyperelastic bodies including non-zero prescribed displacements. Struct Multidiscip Optim 47(1):37-48

Krog LA, Olhoff N (1999) Optimum topology and reinforcement design of disk and plate structures with multiple stiffness and eigenfrequency objectives. Comput Struct 72(4-5):535-563

Lund E (1994) Finite element based design sensitivity analysis and optimization. Ph.D. Dissertation. Inst. Mech Engrg. Aalborg University, Denmark

Luo Y, Li M, Kang Z (2017) Optimal topology design for stress-isolation of soft hyperelastic composite structures under imposed boundary displacements. Struct Multidiscip Optim 55(5):1747-1758

Niu B, Yan J, Cheng G (2009) Optimum structure with homogeneous optimum cellular material for maximum fundamental frequency. Struct Multidiscip Optim 39(2):115

Niu F, Xu S, Cheng G (2011) A general formulation of structural topology optimization for maximizing structural stiffness. Struct Multidiscip Optim 43(4):561-572

Pedersen CB, Buhl T, Sigmund O (2001) Topology synthesis of largedisplacement compliant mechanisms. Int J Numer Methods Eng 50(12):2683-2705

Pedersen NL (2000) Maximization of eigenvalues using topology optimization. Struct Multidiscip Optim 20(1):2-11

Pedersen NL, Nielsen AK (2003) Optimization of practical trusses with constraints on eigenfrequencies, displacements, stresses, and buckling. Struct Multidiscip Optim 25(5-6):436-445

Polizzi E (2009) Density-matrix-based algorithm for solving eigenvalue problems. Phys Rev B 79(11):115112

Seyranian A, Lund E, Olhoff N (1994) Multiple eigenvalues in structural optimization problems. Struct Optim 8(4):207-227

Sigmund O (2001a) Design of multiphysics actuators using topology optimization-Part I: One-material structures. Comput Methods Appl Mech Eng 190(49-50):6577-6604
Sigmund O (2001b) Design of multiphysics actuators using topology optimization-Part II: Two-material structures. Comput Methods Appl Mech Eng 190(49-50):6605-6627

Stolpe M (2010) On some fundamental properties of structural topology optimization problems. Struct Multidiscip Optim 41(5):661670

Svanberg K (1987) The method of moving asymptotes-a new method for structural optimization. Int J Numer Methods Eng 24.2:359373

Thore CJ (2016) Multiplicity of the maximum eigenvalue in structural optimization problems. Struct Multidiscip Optim 53(5):961-965

Wallin M, Ivarsson N, Ristinmaa M (2015) Large strain phase-fieldbased multi-material topology optimization. Int J Numer Methods Eng 104(9):887-904

Wallin M, Ivarsson N, Tortorelli D (2018) Stiffness optimization of non-linear elastic structures. Comput Methods Appl Mech Eng 330:292-307

Wang F, Lazarov BS, Sigmund O, Jensen JS (2014) Interpolation scheme for fictitious domain techniques and topology optimization of finite strain elastic problems. Comput Methods Appl Mech Eng 276:453-472

Yoon GH (2010) Maximizing the fundamental eigenfrequency of geometrically nonlinear structures by topology optimization based on element connectivity parameterization. Comput Struct 88(12):120-133

Young LC (2000) Lectures on the calculus of variations and optimal control theory, vol 304. American Mathematical Society, Providence

Publisher's note Springer Nature remains neutral with regard to jurisdictional claims in published maps and institutional affiliations. 